\title{
AN ODD FURSTENBERG-SZEMERÉDI THEOREM AND QUASI-AFFINE SYSTEMS
}

\author{
BERNARD HOST AND BRYNA KRA
}

\begin{abstract}
We prove a version of Furstenberg's ergodic theorem with restrictions on return times. More specifically, for a measure preserving system $(X, \mathcal{B}, \mu, T)$, an integer $0 \leq j<k$, and $E \subset X$ with $\mu(E)>0$, we show that there exists $n \equiv j(\bmod k)$ with $\mu\left(E \cap T^{-n} E \cap T^{-2 n} E \cap T^{-3 n} E\right)>0$, so long as $T^{k}$ is ergodic. This result requires a deeper understanding of the limit of some non conventional ergodic averages, and the introduction of a new class of systems, the 'Quasi-Affine Systems'.
\end{abstract}

\section{INTRODUCTION}

1.1. Formulation of the problem. Furstenberg [4] proved Szemerédi's theorem via an ergodic theorem:

Theorem 1 (Furstenberg). Let $(X, \mathcal{B}, \mu, T)$ be a measure preserving system and let $\ell \in \mathbb{N}$. Then for any set $E$ with $\mu(E)>0$,

$$
\liminf _{N \rightarrow \infty} \frac{1}{N} \sum_{n=1}^{N} \mu\left(E \cap T^{-n} E \cap T^{-2 n} E \cap \ldots \cap T^{-\ell n} E\right)>0 .
$$

In particular, this implies the existence of $n \geq 1$ so that

$$
\mu\left(E \cap T^{-n} E \cap T^{-2 n} E \cap \ldots \cap T^{-\ell n} E\right)>0 .
$$

We propose here to prove a version of this result for $n$ restricted to a particular congruence class: $n \equiv j(\bmod k)$ for a given $k \geq 2$ and $0 \leq j<k$. For $j=0$, the result follows immediately from Theorem 1 by considering $T^{k}$ instead of $T$ and so we focus on the case $0<j<k$. The case $k=2, j=1$ explains "odd" in the title of this paper.

When $T$ is ergodic and $k$ is prime, an obvious necessary condition is that $T^{k}$ be ergodic. If not, there exists a set $E$ with $\mu(E)>0$ and $\mu\left(E \cap T^{-n} E\right)=0$ for all integers $n$ that are not multiples of $k$.

\subsection{Statement of Results. We prove:}

Theorem 2. Let $(X, \mathcal{B}, \mu, T)$ be a measure preserving system. Assume that $T^{k}$ is ergodic for some integer $k \geq 2$ and let $E$ be a set with $\mu(E)>0$. Let $0 \leq j<k$. Then there exists $n \equiv j(\bmod k)$ so that

$$
\mu\left(E \cap T^{-n} E \cap T^{-2 n} E \cap T^{-3 n} E\right)>0 .
$$

The proof of this theorem relies on the stronger result:

1991 Mathematics Subject Classification. Primary 28D05, Secondary 05A17.

This work was partially carried out while the second author was visiting the Université de Marne la Vallée, supported by NSF grant 9804651. 
Theorem 3. Let $(X, \mathcal{B}, \mu, T)$ be a measure preserving system and assume that $T^{k}$ is ergodic for some integer $k \geq 2$. Let $a_{1}, a_{2}, a_{3}$ be non-zero distinct integers and let $f_{1}, f_{2}, f_{3} \in L^{\infty}(\mu)$. Then the limits

$$
\lim _{N \rightarrow \infty} \frac{1}{N} \sum_{n=1}^{N} \prod_{i=1}^{3} f_{i}\left(T^{k a_{i} n} x\right) \text { and } \lim _{N \rightarrow \infty} \frac{1}{N} \sum_{n=1}^{N} \prod_{i=1}^{3} f_{i}\left(T^{a_{i} n} x\right)
$$

exist in $L^{2}(X)$ and are equal.

The existence of the limits follows from the results of [6]. However, in [6] we were only interested in a short and elementary proof of the existence. Equality of the limits is more intricate and needs a precise evaluation of the limits; this is the main result of this paper. We hope that a deeper understanding can be used for further generalizations. here:

The proof of Theorem 2 is immediate, once we have Theorem 3, and we give it

Proof. (Of Theorem 2, assuming Theorem 3.) For $i=1,2,3$ set $a_{i}=i$ and $f_{i}=$ $\mathbf{1}_{T^{-i j} E}$. Taking the integral over $E$ of the limits in Theorem 3, we have

$$
\lim _{N \rightarrow \infty} \frac{1}{N} \sum_{n=1}^{N} \mu\left(\bigcap_{i=0}^{3} T^{-i(k n+j)} E\right)=\lim _{N \rightarrow \infty} \frac{1}{N} \sum_{n=1}^{N} \mu\left(\bigcap_{i=0}^{3} T^{-i(n+j)} E\right) .
$$

However, this last limit equals

$$
\lim _{N \rightarrow \infty} \frac{1}{N} \sum_{n=1}^{N} \mu\left(\bigcap_{i=0}^{3} T^{-i n} E\right)
$$

which is positive by Theorem 1 .

Question 1. Theorem 1 has a combinatorial interpretation, namely Szemerédi's Theorem. Does Theorem 2 have a combinatorial interpretation?

While we are able to prove the theorem for arithmetic progressions of length four, we do not know if such a result holds more generally.

Question 2. Are results like Theorem 3 and Theorem 2 valid for more terms?

1.3. Strategy. An important ingredient in proving Theorem 1 is a deeper understanding of Conze-Lesigne averages

$$
\frac{1}{N} \sum_{n=1}^{N} f_{1}\left(T^{a_{1} n} x\right) f_{2}\left(T^{a_{2} n} x\right) f_{3}\left(T^{a_{3} n} x\right),
$$

where $f_{1}, f_{2}, f_{3} \in L^{\infty}(\mu)$ and $a_{1}, a_{2}, a_{3}$ are distinct non-zero integers.

The convergence in $L^{2}(\mu)$ of such averages is the main result of [6], generalizing previous results of Conze-Lesigne [3] to the totally ergodic case. Related results have been proved by other authors [5].

We need here more precise information about the limit, and in particular we have to describe how it changes when $k a_{1}, k a_{2}, k a_{3}$ are substituted for $a_{1}, a_{2}, a_{3}$ respectively, assuming the ergodicity of $T^{k}$. The methods of Host and Kra [6], Furstenberg and Weiss [5] and Conze and Lesigne [3] cannot be applied here, as they rely on a change of the initial system. Although convergence in the new system implies convergence in the original one, the essential property of ergodicity of $T^{k}$ 
can be lost in the change. In general, the conclusion of Theorem 3 is not valid for the modified system. Here we work with factors of the original system and so bypass this difficulty.

A main tool in the proofs is the notion of characteristic factor. In our context of three linear terms (of the form (1)) the general definition given in Furstenberg and Weiss [5] can be written as:

Definition 1. A factor $(Y, \mathcal{D}, \nu, T)$ of $(X, \mathcal{B}, \mu, T)$ is characteristic for $\ell$ linear terms if for all distinct, non-zero integers $a_{1}, a_{2}, \ldots, a_{\ell}$ and any $f_{1}, f_{2}, \ldots, f_{\ell} \in$ $L^{\infty}(\mu)$ we have:

$$
\lim _{N \rightarrow \infty} \frac{1}{N} \sum_{n=1}^{N}\left(\prod_{i=1}^{\ell} f_{i}\left(T^{a_{i} n} x\right)-\prod_{i=1}^{\ell} \mathbb{E}\left(f_{i} \mid \mathcal{D}\right)\left(T^{a_{i} n} x\right)\right)=0
$$

in $L^{2}(\mu)$.

Throughout most of this article, we are concerned with three linear terms.

We introduce a class of systems, called quasi-affine systems, in Section 4. They are closely related to the nilpotent manifolds introduced by Conze and Lesigne [3] and lated studied by Rudolph [10]. However, they are considered from a different and, we hope, a simpler point of view. These systems are extensions of their Kronecker factors by a compact abelian group, with an additional property. We show that:

Theorem 4. An ergodic system $(X, \mathcal{B}, \mu, T)$ has a characteristic factor for three linear terms which is a quasi-affine system. In particular, this characteristic factor is a compact abelian group extension of its Kronecker factor.

1.4. Outline of the Paper. In Section 2, we prove an odd ergodic Roth theorem, meaning a convergence theorem for progressions of length three restricted to a particular congruence class. The result follows easily from a theorem of Furstenberg [4] that explicitly describes the limit of expressions of the form

$$
\lim _{N \rightarrow \infty} \frac{1}{N} \sum_{n=1}^{N} f_{1}\left(T^{a_{1} n} x\right) f_{2}\left(T^{a_{2} n} x\right)
$$

for distinct integers $a_{1}, a_{2}$. We give the proof only as a simple example of the use of characteristic factors. In this section we also introduce notations and recall some facts about Kronecker factors. We define the systems $\tilde{Z}$, define a joining of $X$ and state a theorem of Furstenberg and Weiss that. These objects are important in what follows.

In Section 3 we recall without proofs some basic facts about cocycles on rotations. Some other results have been proved elsewhere (see in particular [8]) but are less classical and we give short proofs for completion.

In Section 4 we introduce the notions of a quasi-affine cocycle on an ergodic rotation and of a quasi-affine system. As these cocycles and systems are interesting in themselves we prove more than we actually need for the proof of Theorem 4.

The next two sections are devoted to the proof of Theorem 4 . We progress from simpler to more general systems, proving the result first when $X$ is a group extension of its Kronecker factor, then when it is an isometric extension of its Kronecker factor and then in the general case. Some of the steps follow from results of [5], while others need more work. 
In Section 7, we study the limits of averages (1) for quasi-affine systems. We first compute the "Mackey group" associated to three distinct integers $a_{1}, a_{2}, a_{3}$. Then we use this result to establish the convergence and give an explicit expression of the limit for quasi-affine systems. In particular, we prove Theorem 3 for these systems and in Section 8 we explicitly describe the limit.

1.5. Notations. In the sequel, $Z$ denotes a compact metrizable abelian group, $\mathcal{Z}$ its Borel $\sigma$-agebra and $m$ its Haar measure. For a fixed element $\alpha$ of $Z$, we write $(Z, \alpha)$ for the system $(Z, \mathcal{Z}, m, S)$ where $S: Z \rightarrow Z$ is the transformation $z \mapsto z+\alpha$. Such a system is called a rotation. It is ergodic if and only if $\mathbb{Z} \alpha$ is dense in $Z$.

For an integer $\ell \geq 2$ and distinct integers $a_{1}, a_{2}, \ldots, a_{\ell}$, we write $\tilde{Z}\left(a_{1}, a_{2}, \ldots, a_{\ell}\right)$ for the closed subgroup

$$
\tilde{Z}\left(a_{1}, a_{2}, \ldots, a_{\ell}\right)=\left\{\left(z+a_{1} t, z+a_{2} t, \ldots, z+a_{\ell} t\right): z, t \in Z\right\}
$$

of $Z^{\ell}$. When no confusion is possible, we write $\tilde{Z}$ instead of $\tilde{Z}\left(a_{1}, a_{2}, \ldots, a_{\ell}\right)$. We use $\tilde{m}$ to denote the Haar measure of this group. We write

$$
\tilde{z}=\left(z_{1}, z_{2}, \ldots, z_{\ell}\right)
$$

for an element of $\tilde{Z}$, and

$$
\tilde{\alpha}=\left(a_{1} \alpha, a_{2} \alpha, \ldots, a_{\ell} \alpha\right) \in \tilde{Z} .
$$

The rotation $(\tilde{Z}, \tilde{\alpha})$ will be of a constant use in the sequel.

\section{Preliminaries}

2.1. An Odd-Furstenberg Roth Theorem. In this section, we prove an ergodic version of Roth's Theorem for odd numbers. More specifically, under an assumption of ergodicity, we show the existence of arithmetic progressions of length three with steps taken from a given congruence class.

Theorem 5. Let $(X, \mathcal{B}, \mu, T)$ be a measure preserving system. Assume that $T^{k}$ is ergodic for some integer $k \geq 2$ and let $E$ be a set with $\mu(E)>0$. Let $0 \leq j<k$. Then there exists $n \equiv j(\bmod k)$ so that

$$
\mu\left(E \cap T^{-n} E \cap T^{-2 n} E\right)>0 .
$$

Theorem 5 follows immediately from Theorem 6, as in Section 1.2. Although we only need the result with $a_{1}=0$, we state it more generally.

Theorem 6. Let $(X, \mathcal{B}, \mu, T)$ be a measure preserving system and assume that $T^{k}$ is ergodic for some $k \geq 2$. Then, for distinct integers $a_{1}, a_{2}, a_{3}$ and $f_{1}, f_{2}, f_{3} \in$ $L^{\infty}(\mu)$ we have

$$
\lim _{N \rightarrow \infty} \frac{1}{N} \sum_{n=1}^{N} \int \prod_{i=1}^{3} f_{i}\left(T^{a_{i} n} x\right) d \mu(x)=\lim _{N \rightarrow \infty} \frac{1}{N} \sum_{n=1}^{N} \int \prod_{i=1}^{3} f_{i}\left(T^{k a_{i} n} x\right) d \mu(x) .
$$

For $f_{1}, f_{2} \in L^{\infty}(\mu)$ and $b_{1}, b_{2}$ distinct integers, Furstenberg [4] proved the convergence in $L^{2}(\mu)$ of the averages

$$
\frac{1}{N} \sum_{n=1}^{N} f_{1}\left(T^{b_{1} n} x\right) f_{2}\left(T^{b_{2} n} x\right)
$$

and gave an explicit expression for the limit. Furstenberg and Weiss [5] reformulated this result using terminology of characteristic factors and we recall their method here. First, using Van der Corput Lemma they showed: 
AN ODD FURSTENBERG-SZEMERÉDI THEOREM AND QUASI-AFFINE SYSTEMS 5

Proposition 1 (Furstenberg and Weiss). Let $(X, \mathcal{B}, \mu, T)$ be an ergodic measure preserving system. The Kronecker factor of $X$ is a characteristic factor for two linear terms.

Let $(Z, \alpha)$ be the Kronecker factor of $X$ and $\pi: X \rightarrow Z$ be the natural projection. The rotation $(Z, \alpha)$ is ergodic.

For $i=1,2$, let $\tilde{f}_{i}$ be the functions on $Z$ defined by $\tilde{f}_{i}(\pi(x))=\mathbb{E}\left(f_{i} \mid \mathcal{Z}\right)(x)$. Using the Fourier series expansion of these functions, we have

$$
\lim _{N \rightarrow \infty} \frac{1}{N} \sum_{n=1}^{N} \tilde{f}_{1}\left(S^{b_{1} n} z\right) \tilde{f}_{2}\left(S^{b_{2} n} z\right)=\int_{Z} \tilde{f}_{1}\left(z+b_{1} t\right) \tilde{f}_{2}\left(z+b_{2} t\right) d m(t)
$$

in $L^{2}(m)$. Proposition 1 means that

$$
\lim _{N \rightarrow \infty} \frac{1}{N} \sum_{n=1}^{N}\left(f_{1}\left(T^{b_{1} n} x\right) f_{2}\left(T^{b_{2} n} x\right)-\tilde{f}_{1}\left(\pi\left(T^{b_{1} n} x\right)\right) \tilde{f}_{2}\left(\pi\left(T^{b_{2} n} x\right)\right)\right)=0
$$

in $L^{2}(\mu)$. Thus the average (3) converges in $L^{2}(\mu)$ to the function

$$
f(x)=\int_{Z} \tilde{f}_{1}\left(\pi(x)+b_{1} t\right) \tilde{f}_{2}\left(\pi(x)+b_{2} t\right) d m(t) .
$$

Now we use this result to prove Theorem 6 .

Proof. (of Theorem 6.) Let $f_{1}, f_{2}, f_{3} \in L^{\infty}(\mu)$ and let $a_{1}, a_{2}, a_{3}$ be distinct integers. Using the convergence established above with $b_{1}=a_{1}-a_{3}$ and $b_{2}=a_{2}-a_{3}$, we get

$$
\lim _{N \rightarrow \infty} \frac{1}{N} \sum_{n=1}^{N} \int \prod_{i=1}^{3} f_{i}\left(T^{a_{i} n} x\right) d \mu(x)=\int_{Z} \int_{Z} \prod_{i=1}^{3} \tilde{f}_{i}\left(z+a_{i} t\right) d m(z) d m(t) .
$$

Substituting $k a_{i}$ for $a_{i}$ we also have

$$
\lim _{N \rightarrow \infty} \frac{1}{N} \sum_{n=1}^{N} \int \prod_{i=1}^{3} f_{i}\left(T^{k a_{i} n} x\right) d \mu(x)=\int_{Z} \int_{Z} \prod_{i=1}^{3} \tilde{f}_{i}\left(z+k a_{i} t\right) d m(z) d m(t) .
$$

Since $T^{k}$ is ergodic, the map $t \mapsto k t$ is onto from $Z$ to $Z$. This implies that the integrals in equations (4) and (5) are equal, and the result is proved.

2.2. The measure $\tilde{\mu}$ on $X^{3}$. Let $a_{1}, a_{2}, a_{3}$ be distinct integers. Write $\tilde{Z}=$ $\tilde{Z}\left(a_{1}, a_{2}, a_{3}\right)$ as defined in equation (2).

We define a measure $\tilde{\mu}$ on $X^{3}$ by setting

$$
\int_{X^{3}} \prod_{i=1}^{3} f_{i}\left(x_{i}\right) d \tilde{\mu}\left(x_{1}, x_{2}, x_{3}\right)=\int_{\tilde{Z}} \prod_{i=1}^{3} \tilde{f}_{i}\left(z_{i}\right) d \tilde{m}\left(z_{1}, z_{2}, z_{3}\right),
$$

where $f_{1}, f_{2}, f_{3}$ are any bounded measurable functions on $X$ and $\tilde{f}_{i}$ is the function on $Z$ defined by $\tilde{f}_{i}(\pi(x))=\mathbb{E}\left(f_{i} \mid \mathcal{Z}\right)(x)$. Thus, the convergence in Equation (4) can be written as

$$
\lim _{N \rightarrow \infty} \frac{1}{N} \sum_{n=1}^{N} \int \prod_{i=1}^{3} f_{i}\left(T^{a_{i} n} x\right) d \mu(x)=\int_{\tilde{Z}} \prod_{i=1}^{3} f_{i}\left(x_{i}\right) d \tilde{\mu}\left(x_{1}, x_{2}, x_{3}\right) .
$$

The measure $\tilde{\mu}$ is invariant under the transformation $\tilde{T}=\left(T^{a_{1}} \times T^{a_{2}} \times T^{a_{3}}\right)$. 
The following result was established in [1]; a proof using the Van der Corput Lemma can be found in [5].

Theorem 7. Let $f_{1}, f_{2}, f_{3}$ be bounded measurable functions on $X$ and assume that the function $f_{1}\left(x_{1}\right) f_{2}\left(x_{2}\right) f_{3}\left(x_{3}\right)$ on $X^{3}$ is orthogonal in $L^{2}(\tilde{\mu})$ to the subspace of $\tilde{T}$-invariant functions. Then

$$
\lim _{N \rightarrow \infty} \frac{1}{N} \sum_{n=1}^{N} f_{1}\left(T^{a_{1} n}\right) f_{2}\left(T^{a_{2} n} x\right) f_{3}\left(T^{a_{3} n} x\right)=0
$$

in $L^{2}(\mu)$.

We use this result several times in determining characteristic factors of $X$.

\section{CocyCles on A ROtATion.}

We begin by recalling some basic facts about cocycles and extensions. As we mainly use compact abelian group extensions of rotations we focus on this case.

Let $(Z, \alpha)$ be a rotation and let $m$ denote the Haar measure of $Z$.

3.1. Cocycles and extensions. Let $G$ be a compact metrizable abelian group, written additively, with a Borel $\sigma$-algebra and Haar measure $m_{G}$. A $G$-valued cocycle is a measurable map $\sigma: Z \rightarrow G$.

A $G$-valued cocycle $\sigma$ is a coboundary if there exists a measurable function $b: Z \rightarrow G$ with $\sigma=b \circ T-b$. Two cocycles are cohomologous if their difference is a coboundary.

Each $G$-valued cocycle $\sigma$ defines an extension $\left(Z \times G, T_{\sigma}\right)$ of $(Z, \alpha)$ as follows: $Z \times G$ is endowed with the product $\sigma$-algebra, with the product measure $m \times m_{G}$, and with the transformation $T_{\sigma}$ given by

$$
T_{\sigma}(z, g)=(z+\alpha, g+\sigma(z)) .
$$

The factor map $Z \times G \rightarrow Z$ is projection onto the first coordinate. For every integer $n$, the transformation $T_{\sigma}^{n}$ is given by $T_{\sigma}^{n}(z, g)=\left(z+n \alpha, g+\sigma^{(n)}(z)\right)$ where the map $\sigma^{(n)}: Z \rightarrow G$ is defined by

$$
\sigma^{(n)}(z)= \begin{cases}\sum_{j=0}^{n-1} \sigma(z+j \alpha) & \text { if } n>0 \\ 1 & \text { if } n=0 \\ -\sum_{j=n}^{-1} \sigma(z+j \alpha) & \text { if } n<0 .\end{cases}
$$

For all $m, n \in \mathbb{Z}$, the functions $\sigma^{(n)}(z)$ satisfy the cocycle relation:

$$
\sigma^{(n+m)}(z)=\sigma^{(n)}(z)+\sigma^{(m)}(z+n \alpha) .
$$

The extensions defined by two cohomologous cocycles are clearly isomorphic.

Assuming that $(Z, \alpha)$ is ergodic, we say that the cocycle $\sigma: Z \rightarrow G$ is ergodic if the system $\left(Z \times G, T_{\sigma}\right)$ is ergodic. We say that $\sigma$ is weakly mixing if $\left(Z \times G, T_{\sigma}\right)$ is ergodic and has $Z$ as its Kronecker factor.

Let $\mathcal{S}^{1}$ be the circle group. A multiplicative cocycle is a measurable map $Z \rightarrow \mathcal{S}^{1}$. For multiplicative cocycles we have the same definitions and properties 
as for $G$-valued cocycles, except that the notations are multiplicative instead of additive.

We make frequent use of the following simple lemma about multiplicative cocycles:

Lemma 1. Let $\rho$ be a multiplicative cocycle and let $\left\{n_{j}\right\}$ a sequence of integers such that $n_{j} \alpha \rightarrow 0$ in $Z$. Then

$$
\left\|\rho^{\left(n_{j}\right)}(z+\alpha)-\rho^{\left(n_{j}\right)}(z)\right\|_{2} \rightarrow 0
$$

as $j \rightarrow \infty$.

Proof. For every $j$ we have

$$
\rho^{\left(n_{j}\right)}(z+\alpha) \overline{\rho^{\left(n_{j}\right)}(z)}=\rho\left(z+n_{j} \alpha\right) \overline{\rho(z)}
$$

by applying the cocycle relation (8). Since translations act on $L^{2}(Z)$ in a continuous way, $\left\|\rho\left(z+n_{j} \alpha\right)-\rho(z)\right\|_{2} \rightarrow 0$ and the result follows.

\subsection{A characterization of multiplicative coboundaries.}

Proposition 2. Let $(Z, \alpha)$ be a rotation and let $\rho: Z \rightarrow \mathcal{S}^{1}$ be a multiplicative cocycle. Then $\rho$ is a coboundary if and only if for any sequence $\left\{n_{j}\right\}$ of integers such that $n_{j} \alpha \rightarrow 0$, we have $\rho^{\left(n_{j}\right)}(z) \rightarrow 1$ in $L^{2}(Z)$.

Proof. We first assume that $\rho$ is a coboundary. Let $b$ be a function of modulus 1 on $Z$ such that $b(z+\alpha) \overline{b(z)}=\rho(z)$. Assume that $n_{j} \alpha \rightarrow 0$. For every $j$ we have $\rho^{\left(n_{j}\right)}(z)=b\left(z+n_{j} \alpha\right) \overline{b(z)}$. As $Z$ acts continuously by translations on $L^{2}(Z)$, we have $b\left(z+n_{j} \alpha\right) \rightarrow b(z)$ in $L^{2}(Z)$ and so $\rho^{\left(n_{j}\right)}(z) \rightarrow 1$ in $L^{2}(Z)$.

Conversely, let $H=\overline{\{n \alpha: n \in \mathbb{Z}\}}$, with the closure is taken in $Z$ and let $m_{H}$ be Haar measure on $H$. $Z$ is endowed with a translation invariant distance and we use $\|$.$\| to denote the distance to 0$. By hypothesis for every $\epsilon>0$ there exists $\delta(\epsilon)>0$ such that for all $n$ with $\|n \alpha\|<\delta(\epsilon)$,

$$
\left\|\rho^{(n)}(z)-1\right\|_{2}<\epsilon .
$$

Assume that $\|n \alpha\|<\delta(\epsilon)$. For every $m \in \mathbb{Z}$, by applying the cocycle relation,

$$
\left\|\rho^{(n+m)}(z)-\rho^{(m)}(z)\right\|_{2}=\left\|\rho^{(n)}(z+m \alpha)-1\right\|_{2}=\left\|\rho^{(n)}(z)-1\right\|_{2}<\epsilon
$$

for almost all $z \in Z$.

Let $\left\{n_{j}\right\}$ be a sequence of integers such that $\left\{n_{j} \alpha\right\}$ converges in $Z$. By Equation (9), the sequence $\left\{\rho^{\left(n_{j}\right)}(z)\right\}$ is a Cauchy sequence in $L^{2}(Z)$ and so converges.

Therefore, there exists a continuous mapping $t \mapsto \phi_{t}($.$) from H$ to $L^{2}(Z)$ such that for all $n \in \mathbb{Z}$,

$$
\phi_{n \alpha}(z)=\rho^{(n)}(z)
$$

for almost all $z \in Z$. When $t=n \alpha$ for an integer $n$, the cocycle relation (8) gives

$$
\phi_{t+\alpha}(z)=\phi_{t}(z) \rho(z+t)
$$

for almost all $z \in Z$. By continuity, for all $t \in H$ Equation (10) holds for almost all $z \in Z$.

Thus, for $m$-almost all $z \in Z$, this equations holds for $m_{H}$-almost all $t$. Fix such a $z$. The map $t \mapsto z+t$ is an isomorphism between the ergodic rotation $\left(H, \alpha, m_{H}\right)$ and the ergodic component of $(Z, \alpha, m)$ supported by $z+H$. Equation (10) means that $\rho$ is a coboundary of this ergodic component. Thus $\rho$ is a coboundary for almost all ergodic components of $(Z, \alpha, m)$ and so is a coboundary of $(Z, \alpha, m)$. 
The same argument as the first part of the above proof gives:

Remark 1. Assume that $\rho: Z \rightarrow \mathcal{S}^{1}$ is a multiplicative coboundary and let $\left\{n_{j}\right\}$ be a sequence of integers such that $\left\{n_{j} \alpha\right\}$ converges in $Z$. Then the sequence $\left\{\rho^{\left(n_{j}\right)}(z)\right\}$ converges in $L^{2}(Z)$.

\subsection{Multiplicative cocycles cohomologous to constants.}

Proposition 3. Let $(Z, \alpha)$ be an ergodic rotation and let $\rho: Z \rightarrow \mathcal{S}^{1}$ be a multiplicative cocycle. The following properties are equivalent:

1. The cocycle $\rho$ is cohomologous to a constant.

2. For every sequence $\left\{n_{j}\right\}$ of integers such that $n_{j} \alpha \rightarrow 0$, there exists a sequence $\left\{c_{j}\right\}$ of constants of modulus 1 such that $c_{j} \rho^{\left(n_{j}\right)}(z) \rightarrow 1$ in $L^{2}(Z)$.

3. For every $t \in Z$ the cocycle $\rho(z+t) \overline{\rho(z)}$ is a coboundary.

4. There exists a Borel subset $A$ of $Z$ with $m(A)>0$ so that $\rho(z+t) \overline{\rho(z)}$ is a coboundary for every $t \in A$.

Proof. 1. $\Longrightarrow$ 2. By hypothesis, there exists a function $b$ of modulus 1 on $Z$ and a constant $c$ of modulus 1 such that $b(z+\alpha) \overline{b(z)}=c \rho(z)$. Assume that $n_{j} \alpha \rightarrow 0$. For each $i, b\left(z+n_{j} \alpha\right) \overline{b(z)}=c^{n_{j}} \rho^{\left(n_{j}\right)}(z)$ and the result follows as in the proof of Proposition 2.

2. $\Longrightarrow$ 3. Fix $t \in Z$ and let $\sigma(z)=\rho(z+t) \overline{\rho(z)}$. Let $\left\{n_{j}\right\}$ be a sequence of integers such that $n_{j} \alpha \rightarrow 0$. There exist constants $c_{j}$ such that $c_{j} \rho^{\left(n_{j}\right)}(z) \rightarrow 1$ in $L^{2}(Z)$. Thus, $c_{j} \rho^{\left(n_{j}\right)}(z+t) \rightarrow 1$ and $\sigma^{\left(n_{j}\right)}(z)=\rho^{\left(n_{j}\right)}(z+t) \overline{\rho^{\left(n_{j}\right)}(z)} \rightarrow 1$ in $L^{2}(Z)$. By Proposition 2, we have property (3).

3. $\Longrightarrow$ 4. Clear.

4. $\Longrightarrow 1$. (This part was proved in [8]) Given $t \in A$, there exists a function $b_{t}(z): Z \rightarrow S^{1}$ such that $\rho(z+t) \overline{\rho(z)}=b_{t}(z+\alpha) \overline{b_{t}(z)}$. The function $b_{t}$ is defined up to multiplication by a constant and by classical methods we can choose it so that $t \mapsto b_{t}$ is a Borel mapping from $A$ to $L^{2}(Z)$. Thus, there exists a Borel function $b(t, z)$ on $A \times Z$ with $b_{t}(z)=b(t, z)$ for almost all $(t, z) \in A \times Z$.

Consider the extension $\left(Z \times \mathcal{S}^{1}, T_{\rho}\right)$ of the rotation $(Z, \alpha)$ defined by the cocycle $\rho$. The function

$$
B((x, t),(y, s)) \mapsto b(x-y, y) \mathbf{1}_{A}(x-y) t \bar{s}
$$

is invariant under $T_{\rho} \times T_{\rho}$ on $\left(Z \times \mathcal{S}^{1}\right)^{2}$. Thus it can be written in the form

$$
\sum_{j} c_{j} f_{j}(x, t) \overline{f_{j}(y, s)},
$$

where the $c_{j}$ are constants and the $f_{j}$ are eigenfunctions of $Z \times \mathcal{S}^{1}$. By considering the Fourier coefficients with respect to the variables $s$ and $t$, each function $f_{j}$ can be written as $f_{j}(x, t)=g_{j}(x) t$. As this is an eigenfunction, $\rho$ is cohomologous to the corresponding eigenvalue.

The same proof as the in the implication $\mathbf{1} \Longrightarrow \mathbf{2}$ in this proposition also shows:

Remark 2. Assume that the cocycle $\rho$ is cohomologous to a constant. Then for every sequence $\left\{n_{j}\right\}$ of integers such that $\left\{n_{j} \alpha\right\}$ converges in $Z$, there exists a sequence $\left\{c_{j}\right\}$ of constants of modulus 1 such that $\left\{c_{j} \rho^{\left(n_{j}\right)}(z)\right\}$ converges in $L^{2}(Z)$. 
3.4. Mackey Groups. Let $G$ be a metrizable compact abelian group. Recall that a character of $G$ is a continuous group homomorphism from $G$ to $\mathcal{S}^{1}$. The characters of $G$ form a discrete group $\widehat{G}$ with the operation of pointwise multiplication. $\widehat{G}$ is called the dual group of $G$.

Some properties of a cocycle $\sigma: Z \rightarrow G$ can be translated into properties of the multiplicative cocycles $\chi \circ \sigma$ for $\chi \in \widehat{G}$. The following result is classical.

Proposition 4. Let $(Z, \alpha)$ be an ergodic rotation and let $\sigma$ be a $G$-valued cocycle on $Z$.

1. $\sigma$ is not ergodic if and only if there exists $\chi \in \widehat{G}, \chi \neq 1$, such that $\chi \circ \sigma$ is a coboundary.

2. $\sigma$ is not weakly mixing if and only if there exists $\chi \in \widehat{G}, \chi \neq 1$, such that $\chi \circ \sigma$ is cohomologous to a constant.

More generally, let $\sigma: Z \rightarrow G$ be a cocycle on the ergodic rotation $(Z, \alpha)$, and let

$$
M^{\perp}=\{\chi \in \widehat{G}: \chi \circ \sigma \text { is a coboundary }\} .
$$

$M^{\perp}$ is a subgroup of $\widehat{G}$ and its annihilator

$$
M=\left\{g \in G: \chi(g)=1 \text { for all } \chi \in M^{\perp}\right\}
$$

is a closed subgroup of $G$, called the Mackey group of $\sigma$. For a study of this notion we refer to [5].

Recall that

$$
M^{\perp}=\{\chi \in \widehat{G}: \chi(g)=1 \text { for every } g \in M\}
$$

and that we can identify $M^{\perp}$ with the dual group of $G / M$. We have:

Proposition 5 (Furstenberg and Weiss). Let $f \in L^{2}(Z \times G)$ be such that for all $\chi \in M^{\perp}$,

$$
\int f(z, g) \overline{\chi(g)} d m_{G}(g)=0
$$

for almost all $z \in Z$. Then $f$ is orthogonal to the space of $T_{\sigma}$-invariant functions.

3.5. The Mackey group $\mathbf{M}\left(a_{1}, a_{2}, a_{3}\right)$. Let $(Z, \alpha)$ be an ergodic rotation, $G$ a compact abelian group and $\sigma: Z \rightarrow G$ a cocycle.

Let $a_{1}, a_{2}, a_{3}$ be three distinct integers. As in Section 1.5, we write

$$
\tilde{Z}=\tilde{Z}\left(a_{1}, a_{2}, a_{3}\right)=\left\{\left(z+a_{1} t, z+a_{2} t, z+a_{3} t\right): z, t \in Z\right\}
$$

and use $\tilde{m}$ to denote the Haar measure of this group. We also write

$$
\tilde{\alpha}=\left(a_{1} \alpha, a_{2} \alpha, a_{3} \alpha\right) \in \tilde{Z}
$$

and let $\tilde{S}$ denote the rotation $\tilde{z} \mapsto \tilde{z}+\tilde{\alpha}$ on $\tilde{Z}$.

We define a mapping $\tilde{\sigma}: \tilde{Z} \rightarrow G^{3}$ by

$$
\tilde{\sigma}(\tilde{z})=\left(\sigma^{\left(a_{1}\right.}\left(z_{1}\right), \sigma^{\left(a_{2}\right)}\left(z_{2}\right), \sigma^{\left(a_{3}\right)}\left(z_{3}\right)\right) .
$$

We consider $\tilde{\sigma}$ as a $G^{3}$-valued cocycle of the rotation $(\tilde{Z}, \tilde{\alpha})$ and we associate to this cocycle a subgroup $M^{\perp}$ of $\widehat{G^{3}}=\widehat{G}^{3}$ and a subgroup $M$ of $G^{3}$ as in Section 3.4. However, as $(\tilde{Z}, \tilde{\alpha})$ is not ergodic, it is a bit more delicate. We follow an argument of [5]. 
We write $\tilde{g}=\left(g_{1}, g_{2}, g_{3}\right)$ for an element of $G^{3}$ and $\tilde{\chi}=\left(\chi_{1}, \chi_{2}, \chi_{3}\right)$ for an element of $\widehat{G^{3}}$. For $\tilde{\chi} \in \widehat{G^{3}}$ we write

$$
\tilde{\chi} \circ \tilde{\sigma}(\tilde{z})=\prod_{i=1}^{3} \chi_{i}\left(\sigma^{\left(a_{i}\right)}\left(z_{i}\right)\right) .
$$

The rotation $(\tilde{Z}, \tilde{\alpha})$ has an obvious ergodic decomposition. For every $z \in Z$, let

$$
\tilde{Z}_{z}=\left\{\left(z+a_{1} t, z+a_{2} t, z+a_{3} t\right): t \in Z\right\}
$$

and let $\tilde{m}_{z}$ be the uniform measure on this set. These sets and measures are invariant under the rotation $\tilde{S}$ and for every $z \in Z$ the system $\left(\tilde{Z}_{z}, \tilde{m}_{z}, \tilde{S}\right)$ is an ergodic rotation. We can write

$$
\tilde{m}=\int_{Z} \tilde{m}_{z} d m(z) .
$$

This ergodic decomposition of $\tilde{m}$ is not the standard ergodic decomposition, because the measures $\tilde{m}_{z}$ are not distinct in general.

For every $z \in Z, \tilde{\sigma}$ is defined $\tilde{m}_{z}$-almost everywhere. Thus we can define

$$
M_{z}^{\perp}=\left\{\tilde{\chi} \in \widehat{G}^{3}: \tilde{\chi} \circ \tilde{\sigma} \text { is a coboundary of }\left(\tilde{Z}_{z}, \tilde{m}_{z}, \tilde{S}\right)\right\} .
$$

We write $\stackrel{\Delta}{\alpha}=(\alpha, \alpha, \alpha) \in \tilde{Z}$ and let $\stackrel{\Delta}{S}$ denote the rotation $\tilde{z} \mapsto \tilde{z}+\stackrel{\Delta}{\alpha}$ on $\tilde{Z}$. For every $z, \stackrel{\Delta}{S}$ is an isomorphism from the system $\left(\tilde{Z}_{z}, \tilde{m}_{z}, \tilde{S}\right)$ to the system $\left(\tilde{Z}_{z+\alpha}, \tilde{m}_{z+\alpha}, \tilde{S}\right)$. We define a mapping $\tilde{\sigma}: \tilde{Z} \rightarrow G^{3}$ by

$$
\stackrel{\Delta}{\sigma}(\tilde{z})=\left(\sigma\left(z_{1}\right), \sigma\left(z_{2}\right), \sigma\left(z_{3}\right)\right) .
$$

For every $z \in Z$, using the cocycle relation (8) we have

$$
\tilde{\sigma}(\tilde{z}+\stackrel{\Delta}{\alpha})=\tilde{\sigma}(\tilde{z})+\stackrel{\Delta}{\sigma}(\tilde{z}+\tilde{\alpha})-\stackrel{\Delta}{\sigma}(\tilde{z})
$$

$\tilde{m}_{z}$-almost everywhere. It follows that $M_{z+\alpha}^{\perp}=M_{z}^{\perp}$. But for each $\tilde{\chi} \in \widehat{G}^{3}$, the subset $\left\{z \in Z: \tilde{\chi} \in M_{z}^{\perp}\right\}$ is Borel, and thus has measure 0 or 1 by ergodicity. Therefore, for almost all $z \in Z, M_{z}^{\perp}$ is equal to a constant subgroup $M^{\perp}$ of $\widehat{G}^{3}$.

We can define a subgroup $M$ of $G^{3}$ as in Equation (12), with the relation (13) between $M$ and $M^{\perp}$. Considering each ergodic component of $\tilde{Z}$ separately, it is easy to check that

$$
M^{\perp}=\left\{\tilde{\chi} \in \widehat{G}^{3}: \tilde{\chi} \circ \tilde{\sigma} \text { is a coboundary of }(\tilde{Z}, \tilde{\alpha})\right\}
$$

and that the statement of Proposition 5 remains valid for the extension $\left(\tilde{Z} \times G^{3}, \tilde{S}_{\tilde{\sigma}}\right)$ of $(\tilde{Z}, \tilde{\alpha})$ associated to the cocycle $\tilde{\sigma}$.

In case of ambiguity, we write $M\left(a_{1}, a_{2}, a_{3}\right)^{\perp}$ and $M\left(a_{1}, a_{2}, a_{3}\right)$ instead of $M^{\perp}$ and $M$.

\section{QUASI-AFFINE COCYCLES AND QUASI-AFFINE SYSTEMS.}

\subsection{Quasi-affine cocycles.}

Definition 2. A function $f$ on a compact abelian group $Z$ is said to be affine if $f=c \gamma$, where $c$ is a constant of modulus 1 and $\gamma$ is a character of $Z$.

Proposition 6. Let $(Z, \alpha)$ be an ergodic rotation and let $\rho: Z \rightarrow \mathcal{S}^{1}$ be a multiplicative cocycle. The following properties are equivalent: 
1. For every sequence of integers $\left\{n_{j}\right\}$ with $n_{j} \alpha \rightarrow 0$, there exists a sequence $\left\{w_{j}\right\}$ of affine functions on $Z$ such that $w_{j}(z) \rho^{\left(n_{j}\right)}(z) \rightarrow 1$ in $L^{2}(Z)$.

2. For every $t \in Z$, the cocycle $\rho(z+t) \overline{\rho(z)}$ is cohomologous to a constant.

3. There exists a Borel subset $A$ of $Z$ with $m(A)>0$ so that $\rho(z+t) \overline{\rho(z)}$ is cohomologous to a constant for every $t \in A$.

Definition 3. A multiplicative cocycle $\rho: Z \rightarrow \mathcal{S}^{1}$ satisfying Proposition 6 is said to be a quasi-affine cocycle.

Proof. 1. $\Longrightarrow$ 2. Fix $t \in Z$. Let $\left\{n_{j}\right\}$ be a sequence of integers such that $n_{j} \alpha \rightarrow 0$. There exist constants $c_{j}$ and characters $\gamma_{j}$ such that $\left\|c_{j} \gamma_{j}(z) \rho^{\left(n_{j}\right)}(z)-1\right\|_{2} \rightarrow 0$. Thus $\left\|c_{j} \gamma_{j}(z+t) \rho^{\left(n_{j}\right)}(z+t)\right\|_{2} \rightarrow 0$ and $\left\|\gamma_{j}(t) \rho^{\left(n_{j}\right)}(z+t) \overline{\rho(z)}-1\right\|_{2} \rightarrow 0$. By Proposition 3, the cocycle $z \mapsto \rho(z+t) \overline{\rho(z)}$ is cohomologous to a constant.

2. $\Longrightarrow$ 1. For every $t \in Z$, there exist a constant $c(t)$ and a function $b_{t}(z)$ such that $\rho(z+t) \overline{\rho(z)}=c(t) b_{t}(z+\alpha) \overline{b_{t}(z)}$. The function $b_{t}$ is unique up to multiplication by an affine function and the affine functions form a closed subgroup of functions of modulus 1 on $Z$ with the $L^{2}$ topology. By classical arguments, there is a choice of the functions $b_{t}$ such that the map $t \mapsto b_{t}$ is Borel. With this choice, the function $c(t)$ is Borel on $Z$.

Let $\left\{n_{j}\right\}$ be a sequence of integers such that $n_{j} \alpha \rightarrow 0$. Write $f_{j}(z)=\rho^{\left(n_{j}\right)}(z)$ and $c_{j}(t)=c(t)^{n_{j}}$. For every $t \in Z$, By Proposition 2

$$
\int\left|f_{j}(z+t)-c_{j}(t) f_{j}(z)\right|^{2} d m(z) \rightarrow 0
$$

Integrating with respect to $t$ and taking the Fourier Transform we get

$$
\epsilon_{j}=\sum_{\gamma \in \widehat{Z}}\left|\widehat{f}_{j}(\gamma)\right|^{2}\left(1-\operatorname{Re}\left(\widehat{c_{j}}(\gamma)\right)\right) \rightarrow 0
$$

It follows that there exists $\gamma_{0} \in \widehat{Z}$ with $1-\operatorname{Re}\left(\widehat{c_{j}}\left(\gamma_{0}\right)\right) \leq \epsilon_{j}$. Then $\left|\widehat{c_{j}}(\gamma)\right| \leq 2 \epsilon_{j}$ for $\gamma \neq \gamma_{0}$ and so

$$
\sum_{\gamma \neq \gamma_{0}}\left|\widehat{f}_{j}(\gamma)\right|^{2} \leq \frac{\epsilon_{j}}{1-2 \epsilon_{j}} .
$$

We now have the needed convergence.

2. $\Longrightarrow$ 3. Clear.

3. $\Longrightarrow 2$. The set $H$ of $t \in Z$ so that $\rho(t+z) \overline{\rho(z)}$ is cohomologous to a constant is a Borel subgroup of $Z$. Since $m(H) \geq m(A)>0, H$ is an open subgroup and so is closed. Since $\alpha \in H$, density of the iterates implies that $H=Z$.

4.2. Some properties of quasi-affine cocycles. The product of two quasi-affine cocycles is a quasi-affine cocycle. A cocycle cohomologous to a quasi-affine cocycle is clearly quasi-affine itself. Affine cocycles are clearly quasi-affine and thus so are cocycles which are cohomologous to affine cocycles. However, the converse does not hold. In the appendix we give a counter example, originally due to Furstenberg and Weiss, presented here in our vocabulary.

Using Lemma 1, we immediately have the following corollary:

Corollary 1. Let $(Z, \alpha)$ be an ergodic rotation and let $\rho: Z \rightarrow \mathcal{S}^{1}$ be a cocycle. Let $\left\{n_{j}\right\}$ be a sequence of integers such that $n_{j} \alpha \rightarrow 0,\left\{c_{j}\right\}$ a sequence of constants of modulus 1 , and $\left\{\gamma_{j}\right\}$ a sequence of characters such that $c_{j} \gamma_{j}(z) \rho^{\left(n_{j}\right)}(z) \rightarrow 1$ in $L^{2}(Z)$. Then $\gamma_{j}(\alpha) \rightarrow 1$ as $j \rightarrow \infty$. 
Lemma 2. Let $\rho$ be a multiplicative cocycle, $\left\{n_{j}\right\}$ a sequence of integers so that $\left\{n_{j} \alpha\right\}$ converges (respectively converges to 0 ) in $Z$, and $\left\{c_{j} \gamma_{j}\right\}$ a sequence of affine functions on $Z$ so that $\left\{c_{j} \gamma_{j} \rho^{\left(n_{j}\right)}\right\}$ converges (respectively converges to 1 ) in $L^{2}(Z)$. Then for every integer a,

$$
c_{j}^{a} \gamma_{j}\left(\frac{a(a-1)}{2} n_{j} \alpha\right) \gamma_{j}^{a}(z) \rho^{\left(a n_{j}\right)}(z)
$$

converges (respectively converges to 1 ) in $L^{2}(Z)$.

Proof. For every integer $m$ we have that

$$
c_{j} \gamma_{j}\left(m n_{j} \alpha\right) \gamma_{j}(z) \rho^{\left(n_{j}\right)}\left(z+m n_{j} \alpha\right)
$$

converges (respectively converges to 1 ) in $L^{2}(Z)$. Taking the product for $m$ between 0 and $a$ we have the statement of the lemma.

Lemma 3. Let $\rho$ be a quasi-affine cocycle and let $\left\{n_{j}\right\}$ be a sequence of integers so that the sequence $\left\{n_{j} \alpha\right\}$ converges in $Z$. Then there exists a sequence $\left\{\omega_{j}\right\}$ of affine functions so that the sequence $\left\{\omega_{j}(z) \rho^{\left(n_{j}\right)}(z)\right\}$ converges in $L^{2}(Z)$.

Proof. Choose a translation invariant distance on $Z$ and write $\|z\|$ for the distance of $z \in Z$ to 0 . By the first property of quasi-affine cocycles in Proposition 6 , for every $\epsilon>0$ there exists $\delta(\epsilon)>0$ such that for every $n \in \mathbb{Z}$ with $\|n \alpha\|<\delta$, there exists an affine function $\omega$ with $\left\|1-\omega(z) \rho^{\left(n_{j}\right)}(z)\right\|_{2}<\epsilon$.

Assume that $\left\{n_{j} \alpha\right\}$ converges in $Z$. For every $\epsilon>0$ and for $i, j$ sufficiently large we have $\left\|\left(n_{i}-n_{j}\right) \alpha\right\|<\delta(\epsilon)$. Thus

$$
\left\|\omega\left(z+n_{j} \alpha\right) \rho^{\left(n_{i}\right)}(z)-\rho^{\left(n_{j}\right)}(z)\right\|_{2}=\left\|1-\omega(z) \rho^{\left(n_{i}-n_{j}\right)}(z)\right\|_{2}<\epsilon
$$

and the function $\omega\left(z+n_{j} \alpha\right)$ is affine. Using this, we can construct inductively a sequence $\left\{\omega_{j}\right\}$ of affine functions so that $\left\{\omega_{j} \rho^{\left(n_{j}\right)}\right\}$ is a Cauchy sequence in $L^{2}(Z)$.

Lemma 4. Let $\rho: Z \rightarrow \mathcal{S}^{1}$ be a quasi-affine cocycle. Then either $\rho$ is weakly mixing or $\rho$ is cohomologous to a constant.

Proof. Assume that the quasi-affine cocycle $\rho$ is not weakly mixing. Then there exists a non-zero integer $p$ such that $\rho^{p}$ is cohomologous to a constant.

Let $\left\{n_{j}\right\}$ be a sequence of integers such that $n_{j} \alpha \rightarrow 0$. There exists a sequence $\left\{c_{j} \gamma_{j}\right\}$ of affine functions such that $c_{j} \gamma_{j} \rho^{\left(n_{j}\right)} \rightarrow 1$ in $L^{2}(Z)$ and so $c_{j}^{p} \gamma_{j}^{p} \rho^{p\left(n_{j}\right)} \rightarrow 1$ in $L^{2}(Z)$ as well. Since $\rho^{p}$ is cohomologous to a constant, by Proposition 3 there exists a sequence $\left\{d_{j}\right\}$ of constants such that $d_{j} \rho^{p\left(n_{j}\right)} \rightarrow 1$ in $L^{2}(Z)$. Thus $c_{j}^{p} \gamma_{j}^{p} \overline{d_{j}} \rightarrow 1$ in $L^{2}(Z)$ and for $j$ sufficiently large, $\gamma_{j}^{p}=1$. In particular $\gamma_{j}(\alpha)^{p}=1$. By Corollary 1 , $\gamma_{j}(\alpha) \rightarrow 1$. It follows that for $j$ sufficiently large we have $\gamma_{j}(\alpha)=1$. By the density of $\mathbb{Z} \alpha$ in $Z, \gamma_{j}=1$.

By Proposition 3, $\rho$ is cohomologous to a constant.

4.3. A stability result. By Proposition 3, a multiplicative cocycle $\rho$ is cohomologous to a constant if and only if the cocycle $\rho(z+t) \overline{\rho(z)}$ is a coboundary for all $t$. By definition, $\rho$ is quasi-affine if and only if $\rho(z+t) \overline{\rho(z)}$ is cohomologous to a constant for all $t \in Z$. The next result shows that the same method does not produce more general cocycles. 
Proposition 7. Let $\rho$ be a multiplicative cocycle on $Z$ and let $A$ be a Borel subset of $Z$ with $m(A)>0$ so that for every $s \in A$, the cocycle $\rho(z+s) \overline{\rho(z)}$ is quasi-affine. Then $\rho$ is quasi-affine.

Proof. Let $\left\{n_{j}\right\}$ a sequence of integers so that $n_{j} \alpha \rightarrow 0$ in $Z$. For $j \in \mathbb{N}$ and $s \in Z$ we define

$$
\eta_{j}(s)=\inf \left(\left\|\rho^{\left(n_{j}\right)}(z+s)-c \gamma(z) \rho^{\left(n_{j}\right)}(z)\right\|_{2}\right)
$$

where the infimum is taken over all constants $c$ of modulus 1 and all characters $\gamma \in \widehat{Z}$.

We begin with some simple observations about $\eta_{j}$. By hypothesis, $\eta_{j}(s) \rightarrow 0$ for every $s \in A$. Moreover,

$$
\eta_{j}(\alpha) \leq\left\|\rho^{\left(n_{j}\right)}(z+\alpha)-\rho^{\left(n_{j}\right)}(z)\right\|_{2} .
$$

Thus $\eta_{j}(\alpha) \rightarrow 0$ by Lemma 1 .

Claim 1. For all $s, t \in Z$ and $j \in \mathbb{N}, \eta_{j}(s+t) \leq \eta_{j}(s)+\eta_{j}(t)$.

Proof. Fix $j, s$ and $t$. For $\epsilon>0$ there exist constants $c, c^{\prime}$ and characters $\gamma, \gamma^{\prime}$ with

$$
\left\|\rho^{\left(n_{j}\right)}(z+s)-c \gamma(z) \rho^{\left(n_{j}\right)}(z)\right\|_{2}<\epsilon+\eta_{j}(s)
$$

and

$$
\left\|\rho^{\left(n_{j}\right)}(z+t)-c^{\prime} \gamma^{\prime}(z) \rho^{\left(n_{j}\right)}(z)\right\|_{2}<\epsilon+\eta_{j}(t)
$$

Thus,

$$
\left\|\rho^{\left(n_{j}\right)}(z+t+s)-c^{\prime} \gamma^{\prime}(s) \gamma^{\prime}(z) \rho^{\left(n_{j}\right)}(z+s)\right\|_{2}<\epsilon+\eta_{j}(t)
$$

and so

$$
\left\|\rho^{\left(n_{j}\right)}(z+t+s)-c c^{\prime} \gamma^{\prime}(s)\left(\gamma \gamma^{\prime}\right)(z) \rho^{\left(n_{j}\right)}(z)\right\|_{2}<2 \epsilon+\eta_{j}(s)+\eta_{j}(t) .
$$

Thus $\eta_{j}(s+t) \leq 2 \epsilon+\eta_{j}(s)+\eta_{j}(t)$.

Claim 2. $\eta_{j} \rightarrow 0$ uniformly on $Z$.

Proof. Since $\eta_{j} \rightarrow 0$ pointwise on $A$, there exists a Borel subset $B$ of $A$ with $m(B)>0$ so that $\eta_{j} \rightarrow 0$ uniformly on $B$. By Claim $1, \eta_{j} \rightarrow 0$ uniformly on $B+B$. However $B+B$ has non-empty interior in $Z$. By minimality of the rotation $(Z, \alpha)$ there exists an integer $k>0$ such that

$$
Z=\bigcup_{i=0}^{k} i \alpha+(B+B) .
$$

Since $\eta_{j}(\alpha) \rightarrow 0$, by applying Claim 1 again we have $\eta_{j} \rightarrow 0$ uniformly on each set $i \alpha+(B+B)$ and the result follows.

By Claim 2 and again using Lemma 1 , there exists $j_{0}$ such that for all $j>j_{0}$ and for all $s \in Z$

$$
\eta_{j}(s)<\sqrt{2} / 3
$$

and

$$
\left\|\rho^{\left(n_{j}\right)}(z+\alpha)-\rho^{\left(n_{j}\right)}(z)\right\|_{2}<\sqrt{2} / 3
$$


Fix $j>j_{0}$. For two constants $c, c^{\prime}$ of modulus 1 and two distinct characters $\gamma, \gamma^{\prime}$, we have $\left\|c \gamma-c^{\prime} \gamma^{\prime}\right\|_{2}=\sqrt{2}$. Thus, for every $s$ there exists a unique character $\gamma_{j, s}$ and a constant $c$ of modulus 1 so that

$$
\left\|\rho^{\left(n_{j}\right)}(z+s)-c \gamma_{j, s}(z) \rho^{\left(n_{j}\right)}(z)\right\|_{2}<\sqrt{2} / 3 .
$$

From bound (20), $\gamma_{j, \alpha}=1$.

The constant $c$ is not completely determined by Equation (21). Let $c_{j, s}$ be the constant so that the norm in this equation is minimized. Then

$$
\left\|\rho^{\left(n_{j}\right)}(z+s)-c_{j, s} \gamma_{j, s}(z) \rho^{\left(n_{j}\right)}(z)\right\|_{2}=\eta_{j}(s) .
$$

Since translations act on $L^{2}(Z)$ in a continuous way, by the same continuity argument as above we have that the map $s \mapsto \gamma_{j, s}$ is locally constant on $Z$.

As in the proof of Claim 1 , for $s, t \in Z$ we have

$$
\left\|\rho^{\left(n_{j}\right)}(z+s+t)-c_{j, s} c_{j, t} \gamma_{j, t}(s) \gamma_{j, s}(z) \gamma_{j, t}(z)\right\|_{2} \leq \eta_{j}(s)+\eta_{j}(t) .
$$

Thus

$$
\left\|c_{j, s} c_{j, t} \gamma_{j, t}(s) \gamma_{j, s}(z) \gamma_{j, t}(z)-c_{j, s+t} \gamma_{j, s+t}(z)\right\|_{2} \leq \eta_{j}(s)+\eta_{j}(t)+\eta_{j}(s+t)<\sqrt{2}
$$

and so $\gamma_{j, s+t}=\gamma_{j, s} \gamma_{j, t}$.

Therefore the map $s \mapsto \gamma_{j, s}$ is a group homomorphism from $Z$ to $\widehat{Z}$ that is locally constant and so continuous. As $\gamma_{j, \alpha}=1$, by continuity and density we have $\gamma_{j, s}=1$ for all $s \in Z$.

This means that for $j>j_{0}$ and for all $s \in Z$ we have

$$
\left\|1-\overline{c_{j, s}} \rho^{\left(n_{j}\right)}(z+s) \overline{\rho^{\left(n_{j}\right)}}(z)\right\|_{2}=\left\|f_{j}(z+s)-c_{j, s} f_{j}(z)\right\|_{2}=\eta_{j}(s) \rightarrow 0
$$

as $j \rightarrow \infty$.

As this holds for any sequence $\left\{n_{j}\right\}$ of integers with $n_{j} \alpha \rightarrow 0$, by Proposition 3 for any $s$ the cocycle $\rho(z+s) \overline{\rho(z)}$ is cohomologous to a constant. By Proposition 6, $\rho$ is quasi-affine.

4.4. Quasi-affine Systems. We extend now the definition of quasi-affine cocycles to cocycles with values in an arbitrary compact abelian group:

Definition 4. Let $(Z, \alpha)$ be an ergodic rotation and let $G$ be a metrizable compact abelian group. A cocycle $\sigma: Z \rightarrow G$ is quasi-affine if for every $\chi \in \widehat{G}$, the multiplicative cocycle $\chi \circ \sigma$ is quasi-affine.

This definition is consistent with the definition of a multiplicative quasi-affine cocycles given in Definition 3.

Definition 5. An ergodic system is quasi-affine if it is an extension of its Kronecker factor by a quasi-affine cocycle.

Thus a quasi-affine system $X$ can be written as $X=Z \times G$, where $Z$ is the Kronecker factor of $X, G$ is a compact abelian group and the transformation on $X$ is associated as in Section 3.2 to a quasi-affine cocycle $\sigma: Z \rightarrow G$. Since $Z$ is the Kronecker factor of $X$, in particular the cocycle $\sigma$ is weakly mixing.

Lemma 5. Let $Z \times G$ be a quasi-affine system. Then $\widehat{G}$ is torsion-free and so $k G=G$ for every integer $k \neq 0$. 
Proof. If not, there exists an integer $p \geq 2$ and a non-trivial character $\chi \in \widehat{G}$ with $\chi^{p}=1$. Let $\sigma: Z \rightarrow G$ be the cocycle defining the quasi-affine system $Z \times G$. Since $\chi^{p} \circ \sigma=1$, the multiplicative cocycle $\chi \circ \sigma$ is not weakly mixing. As it is quasi-affine, by Lemma 4 it is cohomologous to a constant. Thus $\sigma$ is not weakly mixing, contradiction.

Proposition 8. Let $X$ be an ergodic system. The family of factors of $X$ which are quasi-affine systems admits a largest element.

Proof. Let $Z$ be the Kronecker factor of $X$. The family of factors of $X$ which are extensions of $Z$ by compact abelian groups contains a largest element $X_{a b}$ (see [5]).

Let $Y$ be a factor of $X$ which is a quasi-affine system. The Kronecker factor $Y_{1}$ of $Y$ is a factor of the Kronecker $Z$ factor of $X$. Since $Z$ is an ergodic rotation, $Z$ and $Y$ are relatively independent extensions of $Y_{1}$. Since $Y$ is a compact abelian group extension of $Y_{1}$, the factor of $X$ spanned by $Y$ and $Z$ is a compact abelian group extension of $Z$ and so is a factor of $X_{a b}$. A fortiori, $Y$ is a factor of $X_{a b}$.

Write $X_{a b}=Z \times G$, where $G$ is a compact abelian group and the transformation is associated to a cocycle $\sigma: Z \rightarrow G$. The subset

$$
\Gamma=\{\chi \in \widehat{G}: \chi \circ \sigma \text { is quasi-affine }\}
$$

is a subgroup of $\widehat{G}$. Its annihilator

$$
\Gamma^{\perp}=\{g \in G: \chi(g)=1 \text { for all } \chi \in \Gamma\}
$$

is a closed subgroup of $G$. Write $G_{1}=G / \Gamma^{\perp}$ and let $\sigma_{1}: Z \rightarrow G_{1}$ be the reduction of $\sigma$ modulo $\Gamma^{\perp}$. The system $\left(Z \times G_{1}, T_{\sigma_{1}}\right)$ is obviously a factor of $\left(Z \times G, T_{\sigma}\right)$.

Since the dual group of $G_{1}$ can be identified with $\Gamma$, the cocycle $\sigma_{1}$ is a quasiaffine cocycle, and $\left(Z \times G_{1}, T_{\sigma_{1}}\right)$ is a a quasi-affine system. Its Kronecker factor is $Z$. Moreover, this system is clearly the largest factor of $\left(Z \times G, T_{\sigma}\right)$ which is a quasi-affine system.

It follows that this factor is the largest factor of $X$ which is a quasi-affine system.

Definition 6. For an ergodic measure preserving system, the largest quasi-affine factor is called the maximal quasi-affine factor of $X$ and is denoted by $X_{Q A}$.

We note that if $X$ is weakly mixing, then both $Z$ and $X_{a b}$ are trivial. Therefore so is $X_{Q A}$.

\section{The CASE OF A GROUP EXTENSION}

We now carry out the first step in the proof of Theorem 4. Namely, we show:

Theorem 8. Assume that the ergodic system $X$ is a compact group extension of its Kronecker factor $Z$. Then the maximal quasi-affine factor of $X$ is characteristic for three linear terms.

We need some preliminaries.

5.1. Coboundaries on $Z \times Z$ and quasi-affine cocycles. Throughout this section, $(Z, \alpha)$ denotes an ergodic rotation. 
Proposition 9. Let $\ell$ be a positive integer and let $a_{1}, a_{2}, \ldots, a_{\ell}$ be distinct integers. Assume that and $\rho_{1}, \rho_{2}, \ldots, \rho_{\ell}$ are multiplicative cocycles on $Z$ so that the multiplicative cocycle

$$
\sigma(z, t)=\prod_{i=1}^{\ell} \rho_{i}\left(z+a_{i} t\right)
$$

is a coboundary for the rotation $(z, t) \mapsto(z+\alpha, t)$ on $Z \times Z$. Then for $i=1,2, \ldots, \ell$, the cocycle $\rho_{i}$ is quasi-affine.

Proof. We proceed by induction on $\ell$. For $\ell=1$ the hypothesis immediately implies that $\rho$ is a coboundary and so is a quasi-affine cocycle.

Let $\ell>1$ and assume that the result holds for $\ell-1$ terms and any distinct integers $a_{1}, a_{2}, \ldots, a_{\ell-1}$.

Fix distinct integers $a_{1}, a_{2}, \ldots, a_{\ell}$ and let $\rho_{1}, \rho_{2}, \ldots, \rho_{\ell}$ be as in the hypothesis of the Proposition. Thus there exists a function $b$ of modulus 1 on $Z \times Z$ so that

$$
\prod_{i=1}^{\ell} \rho_{i}\left(z+a_{i} t\right)=b(z+\alpha, t) \overline{b(z, t)}
$$

for almost all $(z, t) \in Z \times Z$.

For $i=1,2, \ldots, \ell$ set $b_{i}=a_{i}-a_{\ell}$ and fix $s \in Z$. Substituting $z-a_{\ell} s$ for $z$ and $t+s$ for $t$ in Equation (24) we have

$$
\prod_{i=1}^{\ell} \rho_{i}\left(z+a_{i} t+b_{i} s\right)=b\left(z-a_{\ell} s+\alpha, t+s\right) \overline{b\left(z-a_{\ell} s, t+s\right)} .
$$

Writing $\rho_{i}^{\prime}(z)=\rho_{i}\left(z+b_{i} s\right) \overline{\rho_{i}(z)}$ and $b^{\prime}(z, t)=b\left(z-a_{\ell} s, t+s\right) \overline{b(z, t)}$ we have

$$
\prod_{i=1}^{\ell-1} \rho_{i}^{\prime}\left(z+a_{i} t\right)=b^{\prime}(z+\alpha, t) \overline{b^{\prime}(z, t)}
$$

as $b_{\ell}=0$. By the inductive hypothesis, for $i=1,2, \ldots, \ell-1$ the cocycle $\rho_{i}^{\prime}$ is quasi-affine. Thus, for all $s \in b_{i} Z$ the cocycle $\rho_{i}(z+s) \overline{\rho_{i}(z)}$ is quasi-affine. Since the subgroup $b_{i} Z$ of $Z$ is open, we have $m\left(b_{i} Z\right)>0$. By Proposition 7 the cocycle $\rho_{i}$ is quasi-affine for $i=1,2, \ldots, \ell-1$. Exchanging the roles played by the indices $\ell$ and $\ell-1$, we also obtain that the cocycle $\rho_{\ell}$ is quasi-affine.

Proposition 10. Let $\ell$ be a positive integer and let $a_{1}, a_{2}, \ldots, a_{\ell}$ be distinct nonzero integers. Assume that $\rho_{1}, \rho_{2}, \ldots, \rho_{\ell}$ are multiplicative cocycles on $Z$ so that the multiplicative cocycle

$$
\sigma(z, t)=\prod_{i=1}^{\ell} \rho_{i}^{\left(a_{i}\right)}\left(z+a_{i} t\right)
$$

is a coboundary for the rotation $(z, t) \mapsto(z, t+\alpha)$ on $Z \times Z$. Then for $i=1,2, \ldots, \ell$ the cocycle $\rho_{i}$ is quasi-affine.

Proof. By hypothesis, there exists a function $b$ of modulus 1 on $Z \times Z$ so that

$$
\prod_{i=1}^{\ell} \rho_{i}^{\left(a_{i}\right)}\left(z+a_{i} t\right)=b(z, t+\alpha) \overline{b(z, t)}
$$

for almost all $(z, t) \in Z \times Z$. Let $h$ be the function on $Z \times Z$ defined by 


$$
h(z, t) \prod_{i=1}^{\ell} \rho_{i}\left(z+a_{i} t\right)=b(z+\alpha, t) \overline{b(z, t)} .
$$

Using the cocycle relation (8) and Equation (25), we have $h(z, t+\alpha)=h(z, t)$ for almost all $(z, t) \in Z \times Z$. Thus there exists a function $\rho_{\ell+1}$ of modulus 1 on $Z$ with $h(z, t)=\rho_{\ell+1}(z)$ for almost all $(z, t)$. Letting $a_{\ell+1}=0$ we have

$$
\prod_{i=1}^{\ell+1} \rho_{i}\left(z+a_{i} t\right)=b(z+\alpha, t) \overline{b(z, t)} .
$$

By Proposition $9, \rho_{i}$ is quasi-affine for $i=1,2, \ldots, \ell$.

5.2. Proof of Theorem 8. We begin with the abelian case.

Proposition 11. Assume that the ergodic system $X$ is an abelian compact group extension of its Kronecker factor $Z$. Then the maximal quasi-affine factor of $X$ is characteristic for three linear terms.

Proof. Let $Z$ be the Kronecker factor of $X$. By assumption, $X=Z \times G$ where $G$ is a compact abelian group and the transformation $T$ is defined by a cocycle $\sigma: Z \rightarrow G$. Write $x=(z, g)$ for an element of $X$. We fix three distinct non-zero integers $a_{1}, a_{2}, a_{3}$ and use the notations $\tilde{Z}=\tilde{Z}\left(a_{1}, a_{2}, a_{3}\right), \tilde{\sigma}$ and $M=M\left(a_{1}, a_{2}, a_{3}\right)$ previously introduced. We use also the notations $\Gamma, G_{0}$ and $G_{1}$ introduced in Section 4.4.

Let $\tilde{\chi}=\left(\chi_{1}, \chi_{2}, \chi_{3}\right) \in \widehat{G}^{3}$ and assume that $\tilde{\chi} \in M^{\perp}$. By Equation (18), $\tilde{\chi} \circ \tilde{\sigma}$ is a coboundary for the rotation $(\tilde{Z}, \tilde{\alpha})$. Thus the function

$$
(z, t) \mapsto \prod_{i=1}^{3} \chi_{i}\left(\sigma^{\left(a_{i}\right)}\left(z+a_{i} t\right)\right)
$$

is a coboundary for the rotation $(z, t) \mapsto(z, t+\alpha)$ on $Z \times Z$. By Proposition 10 , for $i=1,2,3$ the multiplicative cocycle $\chi_{i} \circ \sigma$ is quasi-affine, meaning that $\chi_{i}$ belongs to $\Gamma$. Thus $M^{\perp} \subset \Gamma^{3}$.

We consider averages of the form Equation (1) for some $f_{1}, f_{2}, f_{3} \in L^{\infty}(\mu)$. Assuming that for some $j \in\{1,2,3\}$ we have

$$
\mathbb{E}\left(f_{j} \mid Z \times G_{1}\right)=0
$$

we have to prove that these averages converge to 0 in $L^{2}(X)$.

The assumption of Equation (26) means that for all $\chi \in \Gamma$ we have

$$
\int f_{j}(z, g) \overline{\chi(g)} d m_{G}(g)=0 .
$$

Recall that in our context the system $\left(X^{3}, \tilde{\mu}, \tilde{T}\right)$ introduced in Section 2.2 is the extension of the rotation $(\tilde{Z}, \tilde{\alpha})$ by the cocycle $\tilde{\sigma}: \tilde{Z} \rightarrow G^{3}$. Thus Proposition 5 applies. Writing $(\tilde{z}, \tilde{g})$ for the element $\left(\left(z_{1}, g_{1}\right),\left(z_{2}, g_{2}\right),\left(z_{3}, g_{3}\right)\right)$ of $X^{3}$, we consider the function

$$
F(\tilde{z}, \tilde{g})=\prod_{i=1}^{3} f_{i}\left(z_{i}, g_{i}\right)
$$


as an element of $L^{2}(\tilde{\mu})$. Equation (27) implies that for almost all $\tilde{z} \in \tilde{Z}$

$$
\int F(\tilde{z}, \tilde{g}) \tilde{\chi}(\tilde{g}) d m_{G^{3}}(\tilde{g})=0
$$

for all $\tilde{\chi} \in \Gamma^{3}$ and thus for all $\tilde{\chi} \in M^{\perp}$. By Proposition 5 the function $F$ is orthogonal in $L^{2}(\tilde{\mu})$ to the subspace of $\tilde{T}$-invariant functions. Thus, by Theorem 7 the averages (1) converge to 0 in $L^{2}(X)$.

This means exactly that $Z \times G_{1}$ is a characteristic factor of $X$.

Using Theorem 7, the machinery of Mackey groups and an algebraic trick, Furstenberg and Weiss[5] proved:

Proposition 12. Assume that the ergodic system $X$ is a compact group extension of its Kronecker factor $Z$. Then $X$ has a characteristic factor for three linear terms which is a compact abelian group extension of $Z$.

Theorem 8 follows immediately by combining this result with Proposition 11 .

\section{Proof of Theorem 4}

Furstenberg and Weiss proved:

Proposition 13. Every ergodic system has a characteristic factor for three linear terms which is an isometric extension of its Kronecker factor.

Using this result, Theorem 4 follows immediately from the following Theorem, which we prove in this Section.

Theorem 9. Assume that the ergodic system $X$ is an isometric extension of its Kronecker factor. Then its maximal quasi-affine factor is characteristic for three linear terms.

We now consider a system $(X, T)$ that is an isometric extension of its Kronecker factor. We prefer to change here usual notations and write $(W, \beta)$ for the Kronecker factor of $X$.

By Lemma 7.2 in Furstenberg and Weiss [5], $X$ can be written as $W \times L / N$ where $L$ is a compact group, $N \subset L$ is a closed subgroup, the transformation $T: X \rightarrow X$ is given by

$$
T(w, \ell N) \mapsto(w+\beta, \tau(w) \ell N)
$$

and the cocycle $\tau: W \rightarrow L$ is ergodic. This means that the transformation $T_{\tau}$ : $W \times L \rightarrow W \times L$ given by

$$
T_{\tau}(w, \ell)=(w+\beta, \tau(w) \ell)
$$

is ergodic.

Unfortunately, difficulty arises from the fact that the Kronecker factor of $W \times L$ is not generally equal to the Kronecker factor $(W, \beta)$ of $W \times L / N$. Let $(Z, \alpha)$ denote the Kronecker factor of $W \times L$ and let $p: W \times L \rightarrow Z$ be the natural projection. In particular, we have

$$
p(w+\beta, \tau(w) \ell)=p(w, \ell)+\alpha .
$$

The factor map $W \times L \rightarrow W \times L / N$ induces a factor map $\pi: Z \rightarrow W$ between the Kronecker factors of these systems; $\pi$ is a continuous, onto group homomorphism and $\pi(\alpha)=\beta$ and $\pi(p(w, \ell))=\ell$. 
We summarize with the following diagram:

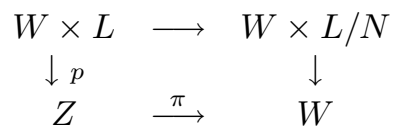

This situation was considered by Furstenberg and Weiss in Lemma 7.3, where they showed that $W \times L$ is isomorphic to a group extension $Z \times M$ of $Z$. We reprove this result, giving an explicit formulation of the isomorphism.

6.1. Understanding the isomorphism. We consider the cocycle $\tau \circ \pi: Z \rightarrow L$. In general, it is not ergodic. Let $M \subset L$ denote its Mackey group. $M$ is a closed subgroup of $L$ characterized up to conjugacy (by an inner automorphism of $L$ ) by the conditions:

1. $\tau \circ \pi$ is cohomologous to a cocycle $\sigma$ with values in $M$

2 . the $M$-valued cocycle $\sigma$ is ergodic.

The first condition means that there exists a measurable mapping $\phi: Z \rightarrow L$ such that

$$
\phi(z+\alpha)^{-1} \tau(\pi(z)) \phi(z)=\sigma(z)
$$

for almost all $z \in Z$. The second condition means that the transformation $T_{\sigma}$ : $(z, m) \mapsto(z+\alpha, \sigma(z) m)$ on $Z \times M$ is ergodic.

By the definition of $T_{\tau}$, Equation (30) and the cohomology equation (31), the mapping

$$
(w, \ell) \mapsto \ell^{-1} \phi(p(w, \ell)) \bmod M
$$

from $W \times L$ to $L / M$ is invariant under $T_{\sigma}$. Thus it is equal almost everywhere to a constant and so there exists $\ell_{0} \in L$ such that $\ell_{0}^{-1} \ell^{-1} \phi(p(w, \ell)) \in M$ for almost all $(w, \ell) \in W \times L$. Substituting $\ell_{0} M \ell_{0}^{-1}$ for $M, \phi(z) \ell_{0}^{-1}$ for $\phi(z)$ and $\ell_{0} \sigma(z) \ell_{0}^{-1}$ for $\sigma(z)$, all the preceding properties remain valid. Moreover,

$$
\phi(p(w, \ell))^{-1} \ell \in M
$$

for almost all $(w, \ell) \in W \times L$.

Lemma 6. The maps $P: W \times L \rightarrow Z \times M$ and $P^{\prime}: Z \times M \rightarrow W \times L$ given by

$$
P(w, \ell)=\left(p(w, \ell), \phi(p(w, \ell))^{-1} \ell\right)
$$

and

$$
P^{\prime}(z, m)=(\pi(z), \phi(z) m) .
$$

are reciprocal isomorphisms between $\left(W \times L, T_{\tau}\right)$ and $\left(Z \times M, T_{\sigma}\right)$.

Proof. It is immediate to check that

$$
T_{\sigma} \circ P=P \circ T_{\tau} ; T_{\tau} \circ P^{\prime}=P^{\prime} \circ T_{\sigma} ; P^{\prime} \circ P=\mathrm{id} .
$$

In particular, $P$ is one to one. The image under $P$ of the measure $m_{W} \times m_{L}$ is a measure on $Z \times M$, invariant under $T_{\sigma}$, and its projection on $Z$ is $m$. Since $\left(Z \times M, T_{\sigma}\right)$ is an ergodic group extension, this measure equals $m \times m_{K}$. The result follows. 
We note that $P$ and $P^{\prime}$ are the isomorphisms constructed in [5].

Right translation of the second coordinate by an element of $L$ is an isomorphism of $W \times L$. Via the isomorphism $P$, this induces an isomorphism of $Z \times M$, which induces an isomorphism of its Kronecker factor $Z$, a rotation. Therefore, for each $u \in L$ there exists $q(u) \in Z$ such that

$$
p(w, \ell u)=p(w, \ell)+q(u)
$$

for almost all $(w, \ell) \in W \times L$.

Lemma 7. Let $K$ be the kernel of $\pi: Z \rightarrow W$. Then $q$ is a continuous group homomorphism from $L$ to $K$ and its kernel is $M$.

Proof. For all $u \in L$ we have $\pi(p(w, \ell))+\pi(q(u))=\pi(p(w, \ell u))=w=\pi(p(w, \ell))$ and so $q(u) \in K$. Moreover, by construction $q: L \rightarrow K$ is a continuous group homomorphism. We now compute its kernel.

The isomorphism $P: W \times L \rightarrow Z \times M$ commutes with right translation of the second coordinate by elements of $M$, since the reciprocal isomorphism $P^{\prime}$ obviously does. Thus, for every $m \in M$, we have $p(w, \ell m)=p(w, \ell)$ for almost all $(w, \ell) \in$ $W \times L$. Therefore, $M \subset \operatorname{ker}(q)$.

Conversely, let $u \in \operatorname{ker}(q)$. By Equations (32) and (33), for almost all $(w, \ell) \in$ $W \times L$ we have modulo $M$

$$
\ell=\phi(p(w, \ell))=\phi(p(w, \ell)+q(u))=\phi(p(w, \ell u))=\ell u
$$

and so $u \in M$. Therefore $\operatorname{ker}(q)=M$.

In particular, we have

Corollary 2. $M$ is normal in $L$ and $L / M$ is abelian.

As $p: W \times L \rightarrow Z$ is onto, it follows from Equation (32) and the definition (33) of $q$ that for all $u \in L$,

$$
\phi(z+q(u))=\phi(z) u \quad \bmod M
$$

for almost all $z \in Z$.

We now show that $q(N)=K$. If not, there exists a character $\chi$ of $Z$ which is trivial on $q(N)$ but not on $K$. Let $f$ be the function on $W \times L$ given by $f(w, \ell)=$ $\chi(p(w, \ell))$. By definition of $q$, for every $n \in N$ we have $f(w, \ell n)=f(w, \ell)$, and $f$ induces a function $g$ on $W \times L / N$. As well, using Equation (30) the function $f$ satisfies $f\left(T_{\tau}(w, \ell)\right)=\chi(\alpha) f(w, \ell)$. Thus $g(T(w, \ell N))=\chi(\alpha) g(w, \ell N)$ and $\chi(\alpha)$ is an eigenvalue of $W \times L / N$. Since $(W, \beta)$ is the Kronecker factor of this system, there exists a character $\psi$ of $W$ such that $\psi(\beta)=\chi(\alpha)$. We get $(\bar{\chi} \cdot \psi \circ \pi)(\alpha)=1$. Since $\mathbb{Z} \alpha$ is dense in $Z, \chi=\psi \circ \pi$ and so $\chi$ is trivial on $K$, a contradiction.

Thus we have $K=q(N) \subset q(L) \subset K$, and $q(N)=q(L)=K$. As $\operatorname{ker}(q)=M$ we get

Lemma 8. $L=M N$.

6.2. A characteristic factor. Recall that $Z \times M$ is an ergodic compact group extension of $Z$, given by the cocyle $\sigma: Z \rightarrow M$, and its Kronecker factor is $Z$. We translate the construction of the maximal quasi-affine factor (see Section 4.4) to this system.

Let $M^{\prime}$ be the commutator subgroup of $M . M^{\prime}$ is a closed normal subgroup of $M$ and $G=M / M^{\prime}$ is abelian. To keep the notations consistent, we notate this 
group additively. Let $\check{\sigma}: Z \rightarrow G$ denote the reduction of $\sigma$ modulo $M^{\prime}$. $\left(Z \times G, T_{\check{\sigma}}\right)$ is the maximal compact abelian group factor $\left(Z \times M, T_{\sigma}\right)_{a b}$ of $\left(Z \times M, T_{\sigma}\right)$.

Let $\Gamma$ be the subgroup of $\widehat{G}$ consisting in characters $\chi \in \widehat{G}$ such that the multiplicative cocycle $\chi \circ \check{\sigma}$ is quasi-affine. $\Gamma^{\perp}$ is the annihilator of $\Gamma$ in $G$ and $G_{1}=G / \Gamma^{\perp}$. The maximal quasi-affine factor of $Z \times M$ is $Z \times G_{1}$, with the transformation given by the cocycle $\sigma_{1}: Z \rightarrow G_{1}$ obtained by reducing the cocycle $\check{\sigma}$ modulo $\Gamma^{\perp}$.

Let $Q$ be the lift in $M$ of the subgroup $\Gamma^{\perp}$ of $G=M / M^{\prime}: Q$ is a normal subgroup of $M$ and we can identify the groups $G_{1}=G / \Gamma^{\perp}$ and $M_{1}=M / Q$. In particular, $\sigma_{1}$ is the reduction of $\sigma$ modulo $Q$.

Lemma 9. For all $\ell \in L$ and all $m \in M$,

$$
\ell^{-1} m \ell m^{-1} \in Q .
$$

Proof. Fix $\ell \in L$. For almost all $z \in Z$ we have $\phi(z)^{-1} \phi(z+q(\ell))=\ell \bmod M$ by Equation (34). Thus there exists a measurable mapping $g: Z \rightarrow M$ such that $\phi(z)^{-1} \phi(z+q(\ell))=g(z) \ell$ for almost all $z \in Z$. By Equation (31), since $q(\ell) \in K=\operatorname{ker}(\pi)$ we get

$$
\sigma(z+q(\ell))=\ell^{-1} g(z+\alpha)^{-1} \sigma(z) g(z) \ell
$$

for almost all $z \in Z$. We now reduce this equation modulo $M^{\prime}$.

Write $\check{g}(z)$ for the reduction of $g(z)$ modulo $M^{\prime}$. Since $M^{\prime}$ is invariant under automorphisms of $L$, it is invariant in particular under the automorphism $m \mapsto \ell^{-1} m \ell$. Thus this automorphism induces an automorphism $j$ of $G: M / M^{\prime}$. Equation (35) gives

$$
\check{\sigma}(z+q(\ell))=j(-\check{g}(z+\alpha)+\check{\sigma}(z)+\check{g}(z)) .
$$

Let $\chi \in \Gamma$, let $\phi$ be the character $\chi \circ j$ of $G$ and let $\psi=\bar{\chi} \phi$. Then

$$
\begin{aligned}
\chi(\check{\sigma}(z+q(\ell))) & =\overline{\phi(\check{g}(z+\alpha))} \phi(\check{g}(z)) \phi(\check{\sigma}(z)) \\
\chi(\check{\sigma}(z+q(\ell))) \overline{\chi(\check{\sigma}(z))} & =\overline{\phi(\check{g}(z+\alpha))} \phi(\check{g}(z)) \psi(\check{\sigma}(z)) .
\end{aligned}
$$

As $\chi \in \Gamma$, by definition the multiplicative cocycle $\chi \circ \check{\sigma}$ is quasi-affine, and by Proposition 6 the multiplicative cocycle

$$
\chi(\check{\sigma}(z+q(\ell))) \overline{\chi(\check{\sigma}(z))}
$$

is cohomologous to a constant. By equation (36), this multiplicative cocycle is also cohomologous to $\psi(\check{\sigma}(z))$ and thus it is cohomologous to a constant. Since $Z$ is the Kronecker factor of $Z \times M$, it is also the Kronecker factor of $Z \times G$. Thus the cocycle $\check{\sigma}$ is weakly mixing and so $\psi=1$. Therefore, $\phi=\chi$ and $\chi \circ j=\chi$.

This means that for all $g \in G, \chi(j(g)-g)=1$. As this holds for all $\chi \in \Gamma$, we get that $j(g)-g \in \Gamma^{\perp}$ for all $g \in G$. Thus $\ell^{-1} m \ell m^{-1} \in Q$ for all $m \in M$ and the Lemma is proved.

Corollary 3. Both $Q$ and $N Q$ are normal subgroups of $L$, and $L / N Q$ is abelian.

Proof. From Lemma 9 and the inclusion $Q \subset M$ it follows immediately that $Q$ is normal in $L$. The Lemma means that $M / Q$ is in the center of $L / Q$. As $L=M N$ we have $L / Q=(M / Q)(N Q / Q)$ and the second statement of the Corollary follows immediately. 
Let $\rho: W \rightarrow L / N Q$ be the reduction modulo $N Q$ of the cocycle $\tau: W \rightarrow L$ and consider $W \times(L / N Q)$ with the transformation $T_{\rho}$. This system is a compact abelian group extension of the rotation $(W, \beta)$. The natural projection $L / N \rightarrow L / N Q$ induces a map $X=W \times L / N \rightarrow W \times L / N Q$, which is clearly a factor map.

Lemma 10. $W \times L / N Q$ is a characteristic factor of $X$ for three linear terms.

Proof. Let $L_{1}=L / Q$ and let $\tau_{1}: W \rightarrow L / Q$ be the reduction of $\tau: W \rightarrow L$ modulo $Q$. Endowed with the transformation $T_{\tau_{1}}, W \times L_{1}$ is a factor of $W \times L$.

The factor $Z \times G_{1}$ of $Z \times M$ is its maximal quasi-affine factor and thus it is a characteristic factor for three linear terms by Theorem 8 . The isomorphism $P^{-1}: Z \times M \rightarrow W \times L$ commutes with the right translations of the second coordinate by elements of $M$, and so in particular by elements of $Q$. Thus it induces an isomorphism of the factor $Z \times G_{1}$ of $Z \times M$ to the factor $W \times L_{1}$ of $W \times L$. Therefore, $W \times L_{1}$ is a characteristic factor of $W \times L$ for three terms.

As usual, we write $\ell N$ for the projection of $\ell \in L$ on $L / N, \ell Q$ for the projection on $L / Q$ and $\ell Q N$ for the projection on $L / Q N$.

Let $a_{1}, a_{2}, a_{3}$ be three distinct, non-zero integers and let $f_{1}, f_{2}, f_{3} \in L^{\infty}(X)$.

Since $Q$ is a normal subgroup of $L$, the conditional expectation of $f_{i}$ on $W \times$ $L / N Q$ can be written

$$
\tilde{f}_{i}(w, \ell N Q)=\int_{Q} f_{i}(w, q \ell N) d m_{Q}(q) .
$$

Let $h_{1}, h_{2}, h_{3} \in L^{\infty}(W \times L)$ be the functions defined by

$$
h_{i}(w, \ell)=f_{i}(w, \ell N) \text {. }
$$

The conditional expectation $\tilde{h}_{i}$ of $h_{i}$ on the factor $W \times L_{1}=W \times L / Q$ of $W \times L$ is:

$$
\begin{gathered}
\tilde{h}_{i}(w, \ell Q)=\int_{Q} h_{i}(w, q \ell) d m_{Q}(q) \\
=\int_{Q} f_{i}(w, q \ell N) d m_{Q}(q) \\
=\tilde{f}_{i}(w, \ell N Q) .
\end{gathered}
$$

As $W \times L_{1}$ is a characteristic factor for three linear terms of $W \times L$ we have

$$
\lim _{N \rightarrow \infty} \frac{1}{N} \sum_{n=1}^{N}\left(\prod_{i=1}^{3} h_{i} \circ T^{a_{i} n}(w, \ell)-\prod_{i=1}^{3} \tilde{h}_{i} \circ T^{a_{i} n}(w, \ell Q)\right)=0
$$

in $L^{2}(W \times L)$. Substituting the values of $h_{i}$ and $\tilde{h}_{i}$ we find that

$$
\lim _{N \rightarrow \infty} \frac{1}{N} \sum_{n=1}^{N}\left(\prod_{i=1}^{3} f_{i} \circ T^{a_{i} n}(w, \ell N)-\prod_{i=1}^{3} \tilde{f}_{i} \circ T^{a_{i} n}(w, \ell N Q)\right)=0
$$

in $L^{2}(W \times L / N)$. This means exactly that $W \times L / Q N$ is a characteristic factor for three linear terms of $W \times L / N$.

We have now assembled the tools to prove Theorem 9:

Proof. (Of Theorem 9.) By Theorem 8, $W \times L / N Q$ has a characteristic factor which is a quasi-affine system. Since $W \times L / N Q$ is a characteristic factor of $X$ for three linear terms, this quasi-affine system is a characteristic factor of $X$ for three linear terms, and the result follows. 


\section{Convergence FOR A QUASI-AFFine System}

Having found a characteristic factor, it now suffices to prove the convergence of the averages (1) and Theorem 3 for quasi-affine systems. Thus we return to quasi-affine systems and compute the Mackey group $M$ introduced in Section 3.5.

Throughout this Section, $X=Z \times G$ is a quasi-affine system. Its Kronecker factor is $(Z, \alpha), G$ is a compact abelian group, and the transformation is associated to the quasi-affine cocycle $\sigma: Z \rightarrow G$. As noted, $\sigma$ is a weakly-mixing cocycle.

Let $a_{1}, a_{2}, a_{3}$ be three non-zero distinct integers. We write

$$
k_{1}^{\prime}=a_{2} a_{3}\left(a_{2}-a_{3}\right), k_{2}^{\prime}=a_{3} a_{1}\left(a_{3}-a_{1}\right), k_{3}^{\prime}=a_{1} a_{2}\left(a_{1}-a_{2}\right) .
$$

Let $A=\operatorname{gcd}\left(k_{1}^{\prime}, k_{2}^{\prime}, k_{3}^{\prime}\right)$ be the greatest common divisor of the $k_{i}^{\prime}$ and let $k_{i}=k_{i}^{\prime} / A$ for $i=1,2,3$. We remark that

$$
\sum_{i=1}^{3} k_{i} a_{i}=\sum_{i=1}^{3} k_{i} a_{i}^{2}=0 \text { and } \operatorname{gcd}\left(k_{1}, k_{2}, k_{3}\right)=1 .
$$

We maintain these notations throughout this section.

7.1. Computation of the Mackey group. In a series of lemmas, we prove the following theorem:

Theorem 10. Assume that the cocycle $\sigma: Z \rightarrow G$ is a weakly mixing quasi-affine cocycle and let $M=M\left(a_{1}, a_{2}, a_{3}\right)$ be the Mackey group associated to the cocycle $\tilde{\sigma}$. Then

$$
\begin{aligned}
M^{\perp} & =\left\{\left(\chi^{k_{1}}, \chi^{k_{2}}, \chi^{k_{3}}\right): \chi \in \widehat{G}\right\} \\
& =\left\{\tilde{\chi} \in \Gamma^{3}: \prod_{i=1}^{3} \chi_{i}^{a_{i}}=\prod_{i=1}^{3} \chi_{i}^{a_{i}^{2}}=1\right\}
\end{aligned}
$$

Corollary 4. Assume that the cocycle $\sigma: Z \rightarrow G$ is a weakly mixing quasi-affine cocycle and let $M=M\left(a_{1}, a_{2}, a_{3}\right)$ be the Mackey group associated to the cocycle $\tilde{\sigma}$. Then

$$
\begin{aligned}
M & =\left\{\left(a_{1} u+a_{1}^{2} v, a_{2} u+a_{2}^{2} v, a_{3} u+a_{3}^{2} v\right): u, v \in G\right\} \\
& =\left\{\left(g_{1}, g_{2}, g_{3}\right): \sum_{i=1}^{3} k_{i} g_{i}=0\right\} .
\end{aligned}
$$

In particular, for every integer $k \geq 1$ we have $M\left(k a_{1}, k a_{2}, k a_{3}\right)=M\left(a_{1}, a_{2}, a_{3}\right)$.

Proof. This follows immediately from Theorem 10 and the relations (12) and (13) between $M$ and $M^{\perp}$.

7.2. The lemmas. Although we omit the hypotheses, we assume throughout this section that the cocycle $\sigma: Z \rightarrow G$ is a weakly mixing quasi-affine cocycle and let $M=M\left(a_{1}, a_{2}, a_{3}\right)$ be the Mackey group associated to the cocycle $\tilde{\sigma}$.

Lemma 11. If $\tilde{\chi} \in M^{\perp}$, then

$$
\prod_{i=1}^{3} \chi_{i}^{a_{i}}=\prod_{i=1}^{3} \chi_{i}^{a_{i}^{2}}=1
$$


Proof. Assume that $n_{j} \alpha \rightarrow 0$ in $Z$. Since $\tilde{\chi} \circ \tilde{\sigma}$ is a coboundary, by Proposition 2

$$
\prod_{i=1}^{3} \chi_{i}\left(\sigma^{\left(n_{j} a_{i}\right)}\left(z_{i}\right)\right) \rightarrow 1
$$

in $L^{2}(\tilde{Z})$. On the other hand, $\chi_{i} \circ \sigma$ is a quasi-affine cocycle for each $i$. Thus there exist constants $c_{j, i}$ and characters $\gamma_{j, i}(z)$ so that

$$
c_{j, i} \gamma_{j, i}(z) \chi_{i}\left(\sigma^{\left(n_{j}\right)}(z)\right) \rightarrow 1
$$

in $L^{2}(Z)$. By Lemma 2 we get

$$
v_{j, i} \gamma_{j, i}^{a_{i}}(z) \chi_{i}\left(\sigma^{\left(n_{j} a_{i}\right)}(z)\right) \rightarrow 1
$$

in $L^{2}(Z)$, where

$$
v_{j, i}=c_{j, i}^{a_{i}} \gamma_{j, i}\left(\frac{a_{i}\left(a_{i}-1\right)}{2} n_{j} \alpha\right)
$$

Thus

$$
\prod_{i=1}^{3} v_{j, i} \gamma_{j, i}^{a_{i}}\left(z_{i}\right) \chi_{i}\left(\sigma^{\left(n_{j} a_{i}\right)}\left(z_{i}\right)\right) \rightarrow 1
$$

in $L^{2}(\tilde{Z})$. Combining Equations (39) and (41), we have

$$
\prod_{i=1}^{3} v_{j, i} \gamma_{j, i}^{a_{i}}\left(z_{i}\right) \rightarrow 1
$$

in $L^{2}(\tilde{Z})$. That is

$$
\int_{Z} \int_{Z}\left|1-\prod_{i=1}^{3} v_{j, i} \gamma_{j, i}\left(a_{i} z+a_{i}^{2} t\right)\right|^{2} d m(z) d m(t) \rightarrow 0 .
$$

It follows that for $j$ sufficiently large

$$
\prod_{i=1}^{3} \gamma_{j, i}^{a_{i}}=\gamma_{j, i}^{a_{i}^{2}}=1
$$

Define

$$
u_{j}=\prod_{i=1}^{3} c_{j, i}^{a_{i}} \text { and } u_{j}^{\prime}=\prod_{i=1}^{3} c_{j, i}^{a_{i}^{2}}
$$

By the convergence in Equation (39), for $j$ sufficiently large,

$$
u_{j} \prod_{i=1}^{3} \chi_{i}^{a_{i}}\left(\sigma^{\left(n_{j}\right)}(z)\right)=\prod_{i=1}^{3} c_{j, i}^{a_{i}} \gamma_{j, i}^{a_{i}}(z) \chi_{i}^{a_{i}} \sigma^{\left(n_{j}\right)}(z) \rightarrow 1
$$

and

$$
u_{j}^{\prime} \prod_{i=1}^{3} \chi_{i}^{a_{i}^{2}}\left(\sigma^{\left(n_{j}\right)}(z)\right)=\prod_{i=1}^{3} c_{j, i}^{a_{i}^{2}} \gamma_{j, i}^{a_{i}^{2}}(z) \chi_{i}^{a_{i}^{2}} \sigma^{\left(n_{j}\right)}(z) \rightarrow 1
$$

in $L^{2}(Z)$. By Proposition 3 the cocycles

$$
\left(\prod_{i=1}^{3} \chi_{i}^{a_{i}}\right) \circ \sigma \text { and }\left(\prod_{i=1}^{3} \chi_{i}^{a_{i}^{2}}\right) \circ \sigma
$$

are cohomologous to constants. Since $\sigma$ is weakly mixing, the result follows. 
Lemma 12. Let $\tilde{\chi} \in M^{\perp}$. Then there exists $\chi \in \widehat{G}$ with $\chi_{i}=\chi^{k_{i}}$ for $i=1,2,3$.

Proof. By Lemma 11 and the definition of the integers $k_{i}^{\prime}$, for every $i, j \in\{1,2,3\}$ we have

$$
\chi_{i}^{k_{j}^{\prime}}=\chi_{j}^{k_{i}^{\prime}} .
$$

By Lemma $5, \widehat{G}$ is torsion free and so

$$
\chi_{i}^{k_{j}}=\chi_{j}^{k_{i}} .
$$

Let $b_{1}, b_{2}, b_{3}$ be integers such that $\sum_{i=1}^{3} b_{i} k_{i}=1$ and $\chi=\prod_{i=1}^{3} \chi_{i}^{b_{i}}$. Then $\chi_{i}=\chi^{k_{i}}$ for $i=1,2,3$.

Lemma 13. For each $\chi \in \widehat{G}$ we have $\left(\chi^{k_{1}}, \chi^{k_{2}}, \chi^{k_{3}}\right) \in M^{\perp}$.

Proof. By the definition of $M^{\perp}$ and use of Proposition 2, it suffices to show that for every sequence of integers $\left\{n_{j}\right\}$ such that $n_{j} \tilde{\alpha} \rightarrow 0$ in $\tilde{Z}$, we have

$$
\prod_{i=1}^{3} \chi^{k_{i}}\left(\sigma^{\left(n_{j} a_{i}\right)}\left(z_{i}\right)\right) \rightarrow 1
$$

in $L^{2}(\tilde{Z})$. Let $a=\operatorname{gcd}\left(a_{1}, a_{2}, a_{3}\right)$. The hypothesis means that $a n_{j} \alpha \rightarrow 0$ in $Z$. Since $\chi \circ \sigma$ is a quasi-affine cocycle, there exist constants $c_{j}$ of modulus 1 and characters $\gamma_{j} \in \widehat{Z}$ such that

$$
c_{j} \gamma_{j}(z) \chi\left(\sigma^{\left(a n_{j}\right)}(z)\right) \rightarrow 1
$$

in $L^{2}(Z)$. For $i=1,2,3$, let $a_{i}^{\prime}=a_{i} / a$ and

$$
v_{j, i}=c_{j}^{a_{j}^{\prime}} \gamma_{j}\left(\frac{a_{i}^{\prime}\left(a_{i}^{\prime}-1\right)}{2} a n_{j} \alpha\right) .
$$

By Lemma 2, for each $i$

$$
v_{j, i} \gamma_{j}^{a_{i}^{\prime}}(z) \chi\left(\sigma^{\left(n_{j} a_{i}\right)}(z)\right) \rightarrow 1
$$

in $L^{2}(Z)$. Thus

$$
\prod_{i=1}^{3} v_{j, i}^{k_{i}} \gamma_{j}^{k_{i} a_{j}^{\prime}}\left(z_{i}\right) \chi^{k_{i}}\left(\sigma^{\left(n_{j} a_{i}\right)}\left(z_{i}\right)\right) \rightarrow 1
$$

in $L^{2}(\tilde{Z})$. We recall that $\sum_{i} k_{i} a_{i}=\sum_{i} k_{i} a_{i}^{2}=0$. It follows immediately that $\prod_{i} v_{j, i}^{k_{i}}=1$. By definition of $\tilde{Z}$, for every $\tilde{z} \in \tilde{Z}$ we have $\sum_{i} k_{i} a_{i}^{\prime} z_{i}=0$ and thus $\prod_{i} \gamma_{j}^{k_{i} a_{i}^{\prime}}\left(z_{i}\right)=1$. Therefore the convergence in equation (43) is exactly the convergence needed for that in equation (42).

7.3. The limit. As above, $X=Z \times G$ is a quasi-affine system, $a_{1}, a_{2}, a_{3}$ are nonzero distinct integers and we maintain the notations of the preceding section.

We study the averages

$$
\frac{1}{N} \sum_{n=1}^{N} f_{1}\left(T^{a_{1} n} x\right) f_{2}\left(T^{a_{2} n} x\right) f_{3}\left(T^{a_{3} n} x\right)
$$

where $f_{1}, f_{2}, f_{3} \in L^{\infty}(X)$. The convergence in $L^{2}(X)$ of these averages and an expression of the limit were established in [6]. We use a similar strategy here, adding in the information gained from the computation of the groups $M$ and $M^{\perp}$ done in Section 7. This gives a more explicit value of the limit. 
Let $\mathcal{M}(Z, G)$ denote the set of maps from $Z$ to $G$, endowed with the topology of convergence in probability.

Proposition 14. There exists a continuous map $t \mapsto \psi_{t}($.$) from Z$ to $\mathcal{M}(Z, G)$ so that for all $n \in \mathbb{Z}$

$$
\psi_{n \alpha}(z)=\sum_{i=1}^{3} k_{i} \sigma^{\left(n a_{i}\right)}(z) .
$$

Proof. Given a sequence $\left\{n_{j}\right\}$ of integers such that $n_{j} \alpha$ converges in $Z$, we have to show that $\sum_{i=1}^{3} k_{i} \sigma^{\left(n a_{i}\right)}(z)$ converges in $\mathcal{M}(Z, G)$. This condition is equivalent to showing that for every character $\chi$ of $G$,

$$
\chi\left(\sum_{i=1}^{3} k_{i} \sigma^{\left(n a_{i}\right)}(z)\right)
$$

converges in $L^{2}(Z)$. Let $\chi \in \widehat{G}$.

Since the system $X=Z \times G$ is quasi-affine, the multiplicative cocycle $\chi \circ \sigma$ is quasi-affine. By Lemma 3 there exists a sequence $\left\{c_{j}\right\}$ in $\mathcal{S}^{1}$ and a sequence $\left\{\gamma_{j}\right\}$ in $\widehat{Z}$ such that $c_{j} \gamma_{j}(z) \chi\left(\sigma^{\left(n_{j}\right)}(z)\right)$ converges in $L^{2}(Z)$. By Lemma 2 , for $i=1,2,3$ the sequence

converges in $L^{2}(Z)$, where

$$
v_{j, i} \gamma_{j}^{a_{j}}(z) \chi\left(\sigma^{a_{i} n_{j}}(z)\right)
$$

$$
v_{j, i}=c_{j}^{a_{i}} \gamma_{j}\left(\frac{a_{j}\left(a_{j}-1\right)}{2} n_{j} \alpha\right) .
$$

Thus

$$
\prod_{i=1}^{3} v_{j, i}^{k_{i}} \gamma^{k_{i} a_{i}}(z) \chi\left(\sum_{i=1}^{3} k_{i} \sigma^{\left(a_{i} n_{j}\right)}(z)\right)
$$

converges in $L^{2}(Z)$. But by the property (37) of the integers $k_{i}$,

$$
\prod_{i=1}^{3} v_{j, i}^{k_{i}}=1 \text { and } \prod_{i=1}^{3} \gamma^{k_{i} a_{i}}(z)=1
$$

for all $z$ and we have the convergence.

Theorem 11. Let $(X, \mathcal{B}, \mu, T)$ be an ergodic measure preserving system and let $f, f_{2}, f_{3} \in L^{\infty}(\mu)$. Let $b_{1}, b_{2}, b_{3}$ be integers such that $\sum_{i} b_{i} k_{i}=1$. Then the averages (1) converge in $L^{2}(X)$ to the function

$$
f(z, g)=\int \prod_{i=1}^{3} f_{i}\left(z+a_{i} t, g+a_{i} u+a_{i}^{2} v+b_{i} \psi_{t}(z)\right) d m_{G}(u) d m_{G}(v) d m(t) .
$$

Proof. By density, it suffices to prove the result when each function $f_{i}$ is of the form

$$
f_{i}(z, g)=\omega_{i}(z) \chi_{i}(g)
$$

for $\omega_{i} \in L^{\infty}(Z)$ and $\chi_{i} \in \widehat{G}$. We write $\tilde{\chi}=\left(\chi_{1}, \chi_{2}, \chi_{3}\right) \in \widehat{G}^{3}$. Using the expression of $M$ given in Corollary 4 , the function $f(z, g)$ in the theorem can be written as

$$
f(z, g)=\int_{Z} \prod_{i=1}^{3} \chi_{i}(g) \omega_{i}\left(z+a_{i} t\right) \chi_{i}\left(b_{i} \psi_{t}(z)\right) d m(t) \int_{M} \tilde{\chi}(\tilde{g}) d m_{M}(\tilde{g}) .
$$


We consider two cases:

1) $\tilde{\chi} \notin M^{\perp}$.

The integral in (45) is clearly equal to 0 and thus the function $f$ given by this equation equals 0 . On the other hand, by the same argument as in the proof of Proposition 11, the averages (1) converge to 0 in $L^{2}(X)$.

2) $\tilde{\chi} \in M^{\perp}$.

For every $\tilde{g} \in M$ we have $\tilde{\chi}(\tilde{g})=1$, Thus the integral in (45) equals 1 . Moreover, by Theorem 10 there exists $\chi \in \widehat{G}$ such that $\chi_{i}=\chi^{k_{i}}$ for $i=1,2,3$. Since $\sum_{i} k_{i} b_{i}=1$, Equation (45) can be written

$$
f(z, g)=\int_{Z} \chi\left(\sum_{i=1}^{3} k_{i} g\right) \prod_{i=1}^{3} \omega_{i}\left(z+a_{i} t\right) \chi\left(\psi_{t}(z)\right) d m(t) .
$$

On the other hand, writing $z=(x, g)$, for every $n$ we have

$$
\prod_{i=1}^{3} f_{i}\left(T^{a_{i} n} x\right)=\chi\left(\sum_{i=1}^{3} k_{i} g\right) \prod_{i=1}^{3} \omega_{i}\left(z+n a_{i} \alpha\right) \chi\left(\sum_{i=1}^{3} \sigma^{\left(n a_{i}\right)}(z)\right) .
$$

This is the value at the point $t=n \alpha$ of the mapping

$$
t \mapsto \chi\left(\sum_{i=1}^{3} k_{i} g\right) \prod_{i=1}^{3} \omega_{i}\left(z+a_{i} t\right) \chi\left(\psi_{t}(z)\right),
$$

a continuous mapping from $Z$ to $L^{2}(Z)$. By unique ergodicity of $(Z, \alpha)$ (see [6]), the average (1) converges in $L^{2}(Z)$ to the integral of this function with respect to the variable $t$, which is exactly the function given by Equation (46).

\section{Conclusion}

We have now assembled the tools needed to prove Theorem 3.

\subsection{The case of quasi-affine systems.}

Proposition 15. Theorem 3 holds for quasi-affine systems.

Proof. Let $f_{1}, f_{2}, f_{3}, a_{1}, a_{2}, a_{3}$ and $k$ be as in the hypothesis of Theorem 3 . We assume that $X$ is a quasi-affine system and maintain the notations of the preceding section. We apply Theorem 11 twice, first with the data $a_{1}, a_{2}, a_{3}$ and then with the data $k a_{1}, k a_{2}, k a_{3}$.

When $k a_{1}, k a_{2}, k a_{3}$ are substituted for $a_{1}, a_{2}, a_{3}$, respectively, the integers $k_{1}, k_{2}, k_{3}$ (as defined in Section 7) remain unchanged. Thus the integers $b_{1}, b_{2}, b_{3}$ appearing in Theorem 11 can be chosen unchanged as well. Let $\psi_{t}$ and $\psi_{t}^{\prime}$ be the mappings associated to the data $a_{1}, a_{2}, a_{3}$ and $k a_{1}, k a_{2}, k a_{3}$ respectively, as in Proposition 14. By construction, for every integer $n$ we have $\psi_{n \alpha}^{\prime}=\psi_{k n \alpha}$. Thus by continuity and density, for all $t \in Z$ we have $\psi_{t}^{\prime}=\psi_{k t}$.

Thus the limit corresponding to $k a_{1}, k a_{2}, k a_{3}$ is equal to

$$
\int \prod_{i=1}^{3} f_{i}\left(z+\left(k a_{i}\right) t, g+\left(k a_{i}\right) u+\left(k a_{i}\right)^{2} v+b_{i} \psi_{k t}(z)\right) d m_{G}(u) d m_{G}(v) d m(t)
$$

at the point $x=(z, g)$. By Lemma 5 the mappings $u \mapsto k u$ and $v \mapsto k^{2} v$ are onto from $G$ to $G$. The ergodicity of $T^{k}$ implies that the mapping $t \mapsto k t$ is onto from $Z$ to $Z$. Thus the integral (47) is equal to the limit corresponding to $a_{1}, a_{2}, a_{3}$ and the proof is complete. 
8.2. The general case. Theorem 3 follows immediately from Proposition 15, Definition 1 of characteristic factors and Theorem 4.

Moreover, we have the following explicit formula for the limit of averages (1) on an arbitrary ergodic system:

Theorem 12. Let $(X, \mathcal{B}, \mu, T)$ be a measure preserving system with Kronecker factor $Z, X_{Q A}=Z \times G$ its maximal quasi-affine factor, and $p: Z \rightarrow Z \times G$ be the natural projection. Let $a_{1}, a_{2}, a_{3}$ be distinct and non-zero integers. There exists a measurable map $(z, t) \mapsto \psi_{t}(z)$ from $Z \times Z$ to $G$ so that for all $f_{1}, f_{2}, f_{3} \in L^{\infty}(X)$,

$\lim _{N \rightarrow \infty} \frac{1}{N} \sum_{n=1}^{N} \prod_{i=1}^{3} f_{i}\left(T^{a_{i} n} x\right)=\int \prod_{i=1}^{3} \mathbb{E}\left(f_{i} \mid X_{Q A}\right)\left(z+a_{i} \alpha, g+a_{i} u+a_{i}^{2} v+\psi_{t}(z)\right) d u d v d t$ in $L^{2}(X)$, where $(z, g)=p(x)$.

\section{APPENDIX: An EXAMPLE OF A QUASI-AFFINE COCYCLE.}

We give here an example, originally due to Furstenberg and Weiss, of a weakly mixing quasi-affine cocycle which is not cohomologous to any affine cocycle. The group $Z$ is $\mathbb{T}^{d}=\mathbb{R}^{d} / \mathbb{Z}^{d}$, where $d$ is an even integer. $\alpha \in \mathbb{T}^{d}$ is chosen so that the rotation $\left(\mathbb{T}^{d}, \alpha\right)$ is ergodic.

Let $A$ be a $d \times d$ matrix with integer entries so that $\operatorname{det}\left(A-A^{*}\right) \neq 0$. Such a matrix exists since $d$ is even. We write $\phi: \mathbb{R}^{d} \times \mathbb{R}^{d} \rightarrow \mathcal{S}^{1}$ for the function defined by

$$
\forall x, y \in \mathbb{R}^{d}, \phi(x, y)=\exp (2 \pi i\langle A x \mid y\rangle)
$$

where $\langle. \mid$.$\rangle is the usual inner product in \mathbb{R}^{d}$.

The cubes $k+[0,1)^{d}, k \in \mathbb{Z}^{d}$, form a partition of $\mathbb{R}^{d}$. We define a function $f$ on $\mathbb{R}^{d}$ by

$$
\forall k \in \mathbb{Z}^{d}, \forall x \in k+[0,1)^{d}, f(x)=\phi(x, k)
$$

we have

$$
\forall x \in \mathbb{R}^{d}, \forall k \in \mathbb{Z}^{d}, f(x+k)=f(x) \phi(x, k)
$$

Let $p: \mathbb{R}^{d} \rightarrow \mathbb{T}^{d}$ be the natural projection and $\beta \in \mathbb{R}^{d}$ be chosen so that $p(\beta)=\alpha$. For $x \in \mathbb{R}^{d}$, set

$$
h(x)=f(x+\beta) \overline{f(x)} \overline{\phi(\beta, x)} .
$$

From Equation (49) it follows that $h(x+k)=h(x)$ for all $x \in \mathbb{R}^{d}$ and all $k \in \mathbb{Z}^{d}$. Thus there exists a function $\rho$ of modulus 1 on $Z$ such that $h=\rho \circ p$, that is

$$
\forall x \in \mathbb{R}^{d}, f(x+\beta) \overline{f(x)} \overline{\phi(\beta, x)}=\rho(p(x)) .
$$

We claim that

Proposition 16. The multiplicative cocycle $\rho: Z \rightarrow \mathcal{S}^{1}$ is weakly mixing, quasiaffine and is not cohomologous to any affine cocycle. 
Proof. Let $\left\{n_{j}\right\}$ be a sequence of integers such that $n_{j} \alpha \rightarrow 0$ in $Z$.

From Equation (50) it follows that, for all $j$ and all $x \in \mathbb{R}^{d}$

$$
\rho^{\left(n_{j}\right)}(p(x))=f\left(x+n_{j} \beta\right) \overline{f(x)} \overline{\phi\left(n_{j} \beta, x\right)} \phi\left(\beta,-\frac{n_{j}\left(n_{j}-1\right)}{2} \beta\right) .
$$

Since $n_{j} \beta \rightarrow 0$ modulo $\mathbb{Z}^{d}$, there exists a sequence $\left\{k_{j}\right\}$ in $\mathbb{Z}^{d}$ and a sequence $\left\{v_{j}\right\}$ converging to 0 in $\mathbb{R}^{d}$ such that $n_{j} \beta=k_{j}+v_{j}$ for all $j$. Using Equation (49), Equation (51) can be written

$$
\begin{aligned}
\rho^{\left(n_{j}\right)}(p(x)) & =f\left(x+v_{j}\right) \overline{f(x)} \overline{\phi\left(v_{j}, x\right)} \overline{c_{j}} \phi\left(x, k_{j}\right) \overline{\phi\left(k_{j}, x\right)} \\
& =f\left(x+v_{j}\right) \overline{f(x)} \overline{\phi\left(v_{j}, x\right)} \overline{c_{j}} \exp \left(2 \pi i\left\langle\left(A-A^{*}\right) x \mid k_{j}\right\rangle\right)
\end{aligned}
$$

where

$$
c_{j}=\overline{\phi\left(v_{j}, k_{j}\right)} \phi\left(\beta, \frac{n(n-1)}{2} \beta\right) .
$$

Since $v_{j} \rightarrow 0$,

$$
\phi\left(x+v_{j}\right) \overline{\phi(x)} \overline{\phi\left(v_{j}, x\right)} \rightarrow 1
$$

in $L^{2}\left([0,1)^{d}\right)$. As the matrix $A-A^{*}$ has integer entries and $k_{j} \in \mathbb{Z}^{d}$, for each $j$ there exists a character $\gamma_{j}$ of $Z=\mathbb{T}^{d}$ with $\overline{\gamma_{j}(p(x))}=\exp \left(2 \pi i\left\langle\left(A-A^{*}\right) x \mid k_{j}\right\rangle\right)$ for all $x \in \mathbb{R}^{d}$. We get

$$
c_{j} \gamma_{j}(z) \rho^{\left(n_{j}\right)}(z) \rightarrow 1
$$

in $L^{2}(Z)$. Thus $\rho$ is quasi-affine.

Assume now that $\rho$ is cohomologous to some affine cocycle $w(z)=c \gamma(z)$ with $c \in \mathcal{S}^{1}$ and $\gamma \in \widehat{Z}$. Let $\left\{n_{j}\right\}$ be a sequence of non zero integers with $n_{j} \alpha \rightarrow 0$ in $Z$, and $k_{j}, v_{j}, \gamma_{j}$ be as above. We also have

$$
\gamma\left(-\frac{n_{j}\left(n_{j}-1\right)}{2} \alpha\right) c^{-n_{j}} \gamma^{-n_{j}}(z) \rho^{\left(n_{j}\right)}(z) \rightarrow 1
$$

in $L^{2}(Z)$, and, comparing with Equation (52), $\gamma_{j}=\gamma^{-n_{j}}$ for all $j$ sufficiently large. For $i, j$ sufficiently large we get $\gamma_{j}^{n_{i}}=\gamma_{i}^{n_{j}}$ and, by definition of $\gamma_{i}$ and $\gamma_{j}$,

$$
\forall u \in \mathbb{R}^{d}, \exp \left(2 \pi i\left\langle\left(A-A^{*}\right) x \mid n_{i} k_{j}-n_{j} k_{i}\right\rangle\right)=1 .
$$

As $\operatorname{det}\left(A-A^{*}\right) \neq 0$, it follows that $k_{i} n_{j}=k_{j} n_{i}$, thus $n_{i} v_{j}=n_{j} v_{i}$. As $v_{j} \rightarrow 0$ we get that for all $i$ sufficiently large we have $v_{i}=0$ thus $n_{i} \alpha=0$, and a contradiction follows.

In particular, $\rho$ is not cohomologous to any constant. By Lemma 4 , it is weakly mixing.

8.3. Remarks. By the same method we could show that $\rho^{m}$ is not cohomologous to an affine cocycle for any integer $m \neq 0$.

Instead of the function $f$ defined by Equation (48), we could use any function satisfying (49). Then the resulting cocycle would be cohomologous to the one we obtained. We could also choose another lift $\beta$ of $\alpha$ in $\mathbb{R}^{d}$, giving rise to the product of $\rho$ and a character. 


\section{REFERENCES}

[1] J.-P. Conze and E. Lesigne. Théorèmes ergodiques pour des mesures diagonales. Bull. Soc. Math. France, 112:143-175, 1984.

[2] J.-P. Conze and E. Lesigne. Sur un théorème ergodique pour des mesures diagonales. $C . R$. Acad. Sci. Paris, Série I, 306:491-493, 1988.

[3] J.-P. Conze and E. Lesigne. Sur un théorème ergodique pour des mesures diagonales. Publications de l'Institut de Recherche de Mathématiques de Rennes, Probabilités, 1987.

[4] H. Furstenberg. Ergodic behavior of diagonal measures and a theorem of Szeméredi on arithmetic progressions. J. d'Analyse Math., 31:204-256, 1977.

[5] H. Furstenberg and B. Weiss. A mean ergodic theorem for $\frac{1}{N} \sum_{n=1}^{n} f\left(T^{n} x\right) g\left(T^{n^{2}} x\right)$. Convergence in Ergodic Theory and Probability, Eds.:Bergelson/March/Rosenblatt, Walter de Gruyter \& Co, Berlin, New York:193-227, 1996.

[6] B. Host and B. Kra. Convergence of Conze-Lesigne Averages. To appear in Erg. Th. E Dyn. Sys.

[7] E. Lesigne. Théorèmes ergodiques pour une translation sur une nilvariété. Erg. Th. E Dyn. Sys., 9(1):115-126, 1989.

[8] E. Lesigne. Résolution d'une équation fonctionelle. Bull. Soc. Math. France, 112:177-196, 1984.

[9] E. Lesigne. Équations fonctionelles, couplages de produits gauches et théorèmes ergodiques pour mesures diagonales. Bull. Soc. Math. France, 121:315-351, 1993.

[10] D.J. Rudolph. Eigenfunctions of $T \times S$ and the Conze-Lesigne algebra. Ergodic Theory and its Connections with Harmonic Analysis, Eds.:Petersen/Salama, Cambridge University Press, New York:369-432, 1995.

Equipe D'analyse et de mathématiques appliquées, Université de Marne la Vallée, 77454 Marne la Vallée CedeX

Department of Mathematics, 231 W. 18th Ave, The Ohio State University, Columbus, Оніо 43210

E-mail address: host@math.univ-mlv.fr

E-mail address: kra@math.ohio-state.edu 